\title{
Offshore Oilfield Development Planning under Uncertainty and Fiscal Considerations
}

\author{
Vijay Gupta ${ }^{1}$ and Ignacio E. Grossmann ${ }^{2}$ \\ Department of Chemical Engineering, Carnegie Mellon University \\ Pittsburgh, PA 15213
}

\begin{abstract}
The objective of this paper is to present a unified modeling framework to address the issues of uncertainty and complex fiscal rules in the development planning of offshore oil and gas fields which involve critical investment and operational decisions. In particular, the paper emphasizes the need to have as a basis an efficient deterministic model that can account for various alternatives in the decision making process for a multi-field site incorporating sufficient level of details in the model, while being computationally tractable for the large instances. Consequently, such a model can effectively be extended to include other complexities, for instance endogenous uncertainties and a production sharing agreements. Therefore, we present a new deterministic MINLP model followed by discussion on its extensions to incorporate generic fiscal rules, and uncertainties based on recent work on multistage stochastic programming. Numerical results on the development planning problem for deterministic as well as stochastic instances are discussed. A detailed literature review on the modeling and solution methods that are proposed for each class of the problems in this context is also presented.
\end{abstract}

Keywords: Multi-period optimization, Planning, Offshore Oil and Gas, Multistage Stochastic, Endogenous, Production Sharing Agreements (PSAs)

\section{Introduction}

The development planning of offshore oil and gas fields has received significant attention in recent years given the new discoveries of large oil and gas reserves in the last decade around the

\footnotetext{
${ }^{1}$ E-mail: vijaygup@andrew.cmu.edu

${ }^{2}$ To whom all correspondence should be addressed. E-mail: grossmann@cmu.edu
} 
world. These have been facilitated by the new technologies available for exploration and production of oilfields in remote locations that are often hundreds of miles offshore. Surprisingly, there has been a net increase in the total oil reserves in the last decade because of these discoveries despite increase in the total demand (BP, Statistical review Report 2011). Therefore, there is currently a strong focus on exploration and development activities for new oil fields all around the world, specifically at offshore locations. However, installation and operation decisions in these projects involve very large investments that potentially can lead to large profits, but also to losses if these decisions are not made carefully.

With the motivation described above, the paper addresses the optimal development planning of offshore oil and gas fields in a generic way and discusses the key issues involved in this context. In particular, a unified modeling framework (Fig. 1) is presented starting with a basic deterministic model that includes sufficient level of detail to be realistic as well as computationally efficient. Moreover, we discuss the extension of the model for incorporating uncertainty based on multistage stochastic programming, and fiscal rules defined by the terms of the contract between oil companies and governments.

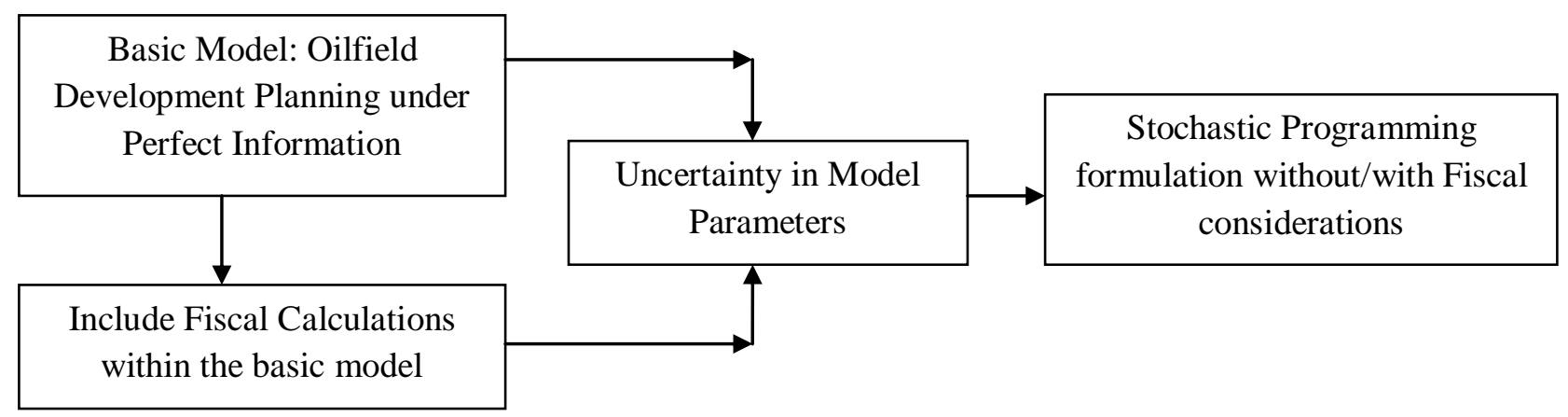

Figure 1: A unified framework for Oilfield Development planning under uncertainty and complex fiscal rules

The planning of offshore oil and gas field development represents a very complex problem and involves multi-billion dollar investments (Babusiaux et al., 2007). The major decisions involved in the oilfield development planning phase are the following:

(a) Selecting platforms to install and their sizes

(b) Deciding which fields to develop and what should be the order to develop them

(c) Deciding which wells and how many are to be drilled in the fields and in what sequence

(d) Deciding which fields are to be connected to which facility 
(e) Determining how much oil and gas to produce from each field

Therefore, there are a very large number of alternatives that are available to develop a particular field or group of fields. However, these decisions should account for the physical and practical considerations, such as the following: a field can only be developed if a corresponding facility is present; nonlinear profiles of the reservoir to predict the actual flowrates of oil, water and gas from each field; limitation on the number of wells that can be drilled each year due to availability of the drilling rigs; long-term planning horizon that is the characteristics of the these projects. Therefore, optimal investment and operations decisions are essential for this problem to ensure the highest return on the investments over the time horizon considered.

By including all the considerations described above in an optimization model, this leads to a large scale multiperiod MINLP problem. The extension of this model to the cases where we explicitly consider the fiscal rules (Van den Heever et al. (2000) and Van den Heever and Grossmann (2001)) and the uncertainties, especially endogenous uncertainty cases (Jonsbraten et al. (1998), Goel and Grossmann (2004, 2006), Goel et al. (2006), Tarhan et al. (2009, 2011) and Gupta and Grossmann (2011)), can lead to a very complex problem to solve. Therefore, an effective model for the deterministic case is essential. On one hand such a model must capture realistic reservoir profiles, interaction among various fields and facilities, wells drilling limitations and other practical trade-offs involved in the offshore development planning, and on the other hand can be used as the basis for extensions that include other complexities, especially fiscal rules and uncertainties as can be seen in Figure 1.

The paper starts with a brief background on the basic structure of an offshore oilfield site and major reservoir features. Next, a review of the various approaches considered in the literature for optimal oilfield development under perfect information is outlined. The key strategic/tactical decisions and details to be included with a generic deterministic model for multi-field site are presented. Given the importance of the explicit consideration of the uncertainty in the development planning, the recent work on the multistage stochastic programming approaches in this context is highlighted. Furthermore, based on the above unified framework and literature review, discussions on the extension of the proposed deterministic model to incorporate uncertainty and generic fiscal rules within development planning are also presented. Numerical results on several examples ranging from deterministic to stochastic cases for this planning problem are reported. 


\section{Background}

\section{(a) Basic elements of an offshore oilfield planning}

The life cycle of a typical offshore oilfield project consists of following five steps:

1) Exploration: This activity involves geological and seismic surveys followed by exploration wells to determine the presence of oil or gas.

2) Appraisal: It involves drilling of delineation wells to establish the size and quality of the potential field. Preliminary development planning and feasibility studies are also performed.

3) Development: Following a positive appraisal phase, this phase aims at selecting the most appropriate development plan among many alternatives. This step involves capitalintensive investment and operations decisions that include facility installations, drilling, sub-sea structures, etc.

4) Production: After facilities are built and wells are drilled, production starts where gas or water is usually injected in the field at a later time to enhance productivity.

5) Abandonment: This is the last phase of an oilfield development project and involves the decommissioning of facility installations and subsea structures associated with the field.

Given that most of the critical investment decisions are usually associated with the development planning phase of the project, this paper focuses on the key decisions during this phase of the project.

An offshore oilfield infrastructure (Fig. 2) consists of various production facilities such as Floating Production, Storage and Offloading (FPSO), Tension Leg platform (TLP), fields, wells and connecting pipelines to produce oil and gas from the reserves. Each oilfield consists of a number of potential wells to be drilled using drilling rigs, which are then connected to the facilities through pipelines to produce oil. There is two-phase flow in these pipelines due to the presence of gas and liquid that comprises oil and water. Therefore, there are three components, and their relative amounts depend on certain parameters like cumulative oil produced.

The field to facility connection involves trade-offs associated to the flowrates of oil and gas, piping costs, and possibility of other fields to connect to that same facility. The number of wells that can be drilled in a field depends on the availability of the drilling rig that can drill a certain number of wells each year. 


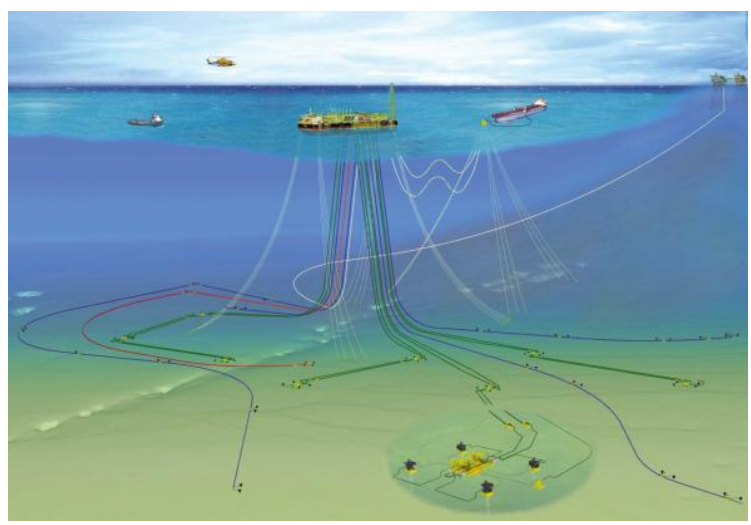

Figure 2: A Complex Offshore Oilfield Infrastructure

The facilities and piping connections in the offshore infrastructure are often in operation over many years. It is therefore important to anticipate future conditions when designing an initial infrastructure or any expansions. This can be accomplished by dividing the planning horizon, for example, 20 years, into a number of time periods with a length of 1 year, and allowing investment and operating decisions in each period, which leads to a multi-period planning problem.

\section{(b) Development planning Problem Specifications}

We assume in this paper that the type of offshore facilities connected to fields to produce oil and gas are FPSOs (Fig. 3). The extension for including Tension Leg Platform (TLP) is straightforward but for simplicity we only consider FPSOs with continuous capacities and ability to expand them in the future. These FPSO facilities cost multi-billion dollars depending on their sizes, and have the capability of operating in remote locations for very deep offshore oilfields (200m-2000m) where seabed pipelines are not cost effective. FPSOs are large ships that can process the produced oil and store it until it is shipped to the onshore site or sales terminal. Processing includes the separation of oil, water and gas into individual streams using separators located at these facilities. Each FPSO facility has a lead time between the construction or expansion decision, and its actual availability. The wells are subsea wells in each field that are drilled using drilling ships. Therefore, there is no need to have a facility present to drill a subsea well. The only requirement to recover oil from it is that the well must be connected to a FPSO facility. 


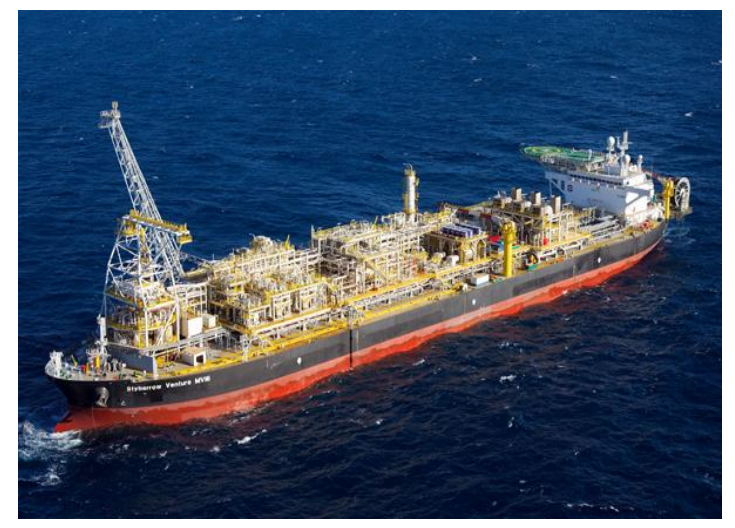

Figure 3: FPSO (Floating Production Storage and Offloading) facility

In this paper, we consider a typical offshore oilfield infrastructure (Figure 4) as a reference to model the problem of oilfield development planning. In particular, given are a set of oil fields $F$ $=\{1,2, \ldots f\}$ available for producing oil using a set of FPSO (Floating, Production, Storage and Offloading) facilities, $F P S O=\{1,2, \ldots f p s o\}$, that can process the produced oil, store and offload it to the other tankers. Each oilfield consists of a number of potential wells to be drilled using drilling rigs, which are then connected to these FPSO facilities through pipelines to produce oil.

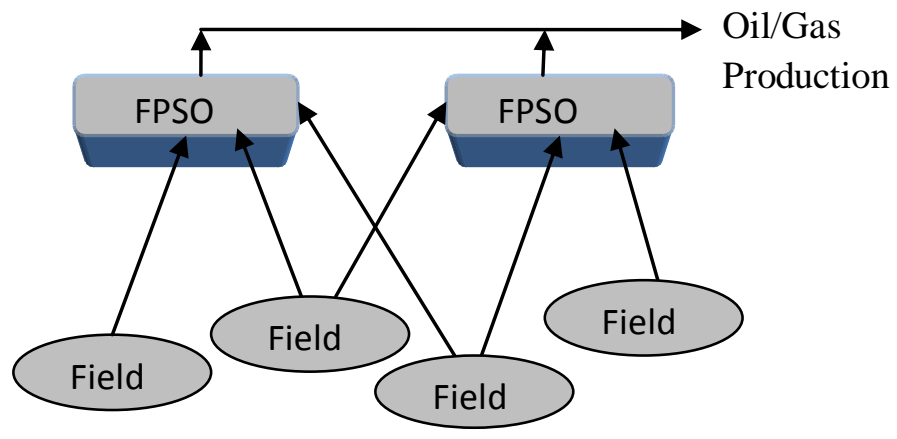

Figure 4: A typical offshore oilfield infrastructure

The location of production facilities and possible field and facility allocation itself is a very complex problem. In this work, we assume that the potential location of facilities and fieldfacility connections are given. In addition, the potential number of wells in each field is also given. Note that each field can be potentially allocated to more than one FPSO facility, but once the particular field-connection is selected, the other possibilities are not considered. Furthermore, each facility can be used to produce oil from more than one field. We assume for simplicity that there is no re-injection of water or gas in the fields. 
The problem considers strategic/tactical decisions to maximize the total NPV of the project under given constraints. The proposed model, as explained in the next section, focuses on the multi-field site presented here and includes sufficient details to account for the various trade-offs involved without going into much detail for each of these fields. However, the proposed model can easily be extended to include various facility types and other details in the oilfield development planning problem.

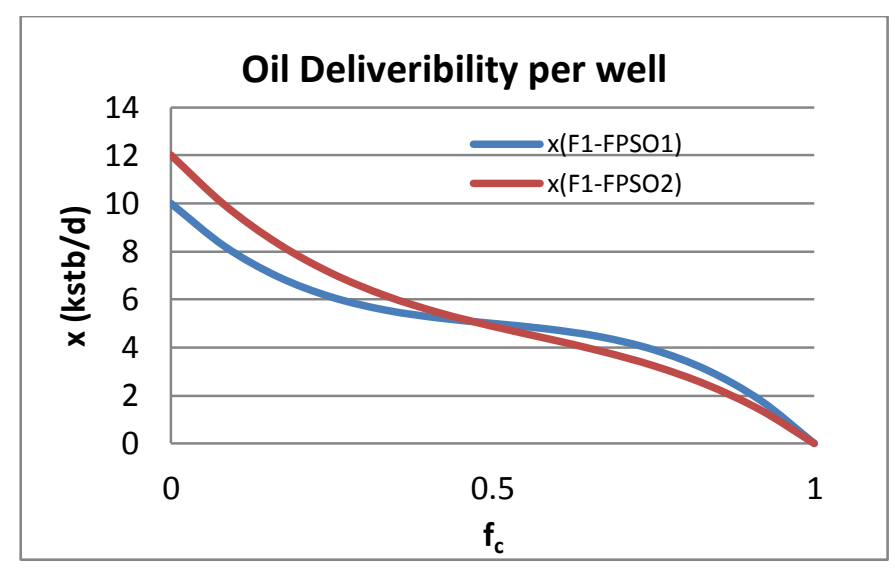

(a) Oil Deliverability per well for field (F1)

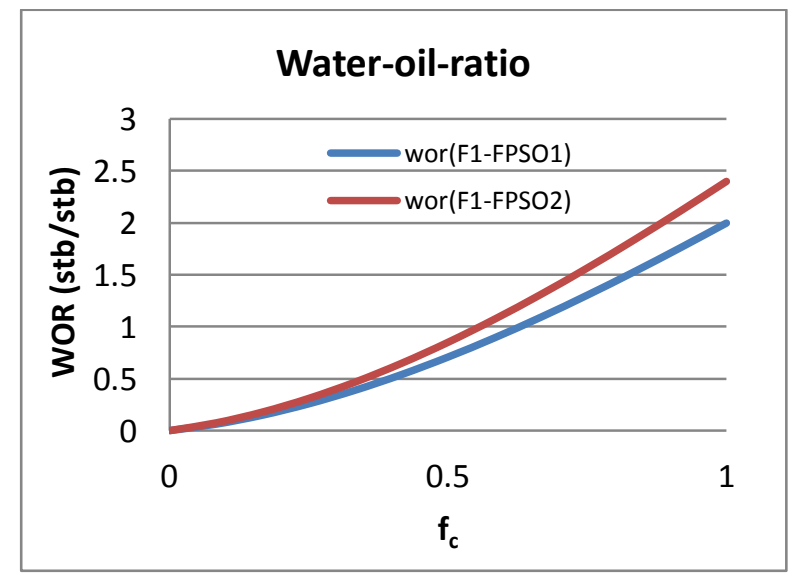

(b) Water to oil ratio for field (F1)

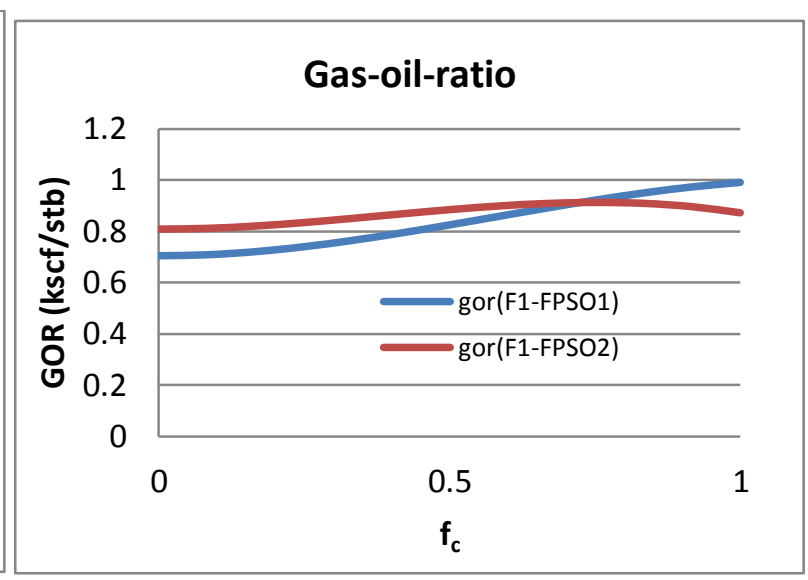

(b) Gas to oil ratio for field (F1)

Figure 5: Nonlinear Reservoir Characteristics for field (F1) for 2 FPSO facilities (FPSO 1 and 2)

When oil is extracted from a reservoir oil deliverability, water-to-oil ratio (WOR) and gas-tooil ratio (GOR) change nonlinearly as a function of the cumulative oil recovered from the reservoir. The initial oil and gas reserves in the reservoirs, as well as the relationships for WOR 
and GOR in terms of fractional recovery $\left(f_{c}\right)$, are estimated from geologic studies. Figures 5 (a) (c) represent the oil deliverability from a field per well, WOR and GOR versus fractional oil recovered from that field.

The maximum oil flowrate (field deliverability) per well can be represented as a $3^{\text {rd }}$ order polynomial equation (a) in terms of the fractional recovery. Furthermore, the actual oil flowrate $\left(x_{f}\right)$ from each of the wells is restricted by both the field deliverability $Q_{f}^{d}$, (b), and facility capacity. We assume that there is no need for enhanced recovery, i.e., no need for injection of gas or water into the reservoir. The oil produced from the wells $\left(x_{f}\right)$ contains water and gas and their relative rates depend on water-to-oil ratio (wor $)$ and gas-to-oil ratio $\left(\right.$ gor $\left._{f}\right)$ that are approximated using $3^{\text {rd }}$ order polynomial functions in terms of fractional oil recovered (eqs. (c)(d)). The water and gas flowrates can be calculated by multiplying the oil flowrate $\left(x_{f}\right)$ with water-to-oil ratio and gas-to-oil ratio as in eqs. (e) and (f), respectively. Note that the reason for considering fractional oil recovery compared to cumulative amount of oil is to avoid numerical difficulties that can arise due to very small magnitude of the polynomial coefficients in that case.

$$
\begin{array}{cc}
Q_{f}^{d}=a_{1, f}\left(f c_{f, t}\right)^{3}+b_{1, f}\left(f c_{f}\right)^{2}+c_{1, f} f c_{f}+d_{1} & \forall f \\
x_{f} \leq Q_{f}^{d} & \forall f \\
\text { wor }_{f}=a_{2, f}\left(f c_{f}\right)^{3}+b_{2, f}\left(f c_{f}\right)^{2}+c_{2, f} f c_{f}+d_{2, f} & \forall f \\
g o r_{f}=a_{3, f}\left(f c_{f}\right)^{3}+b_{3, f}\left(f c_{f}\right)^{2}+c_{3, f} f c_{f}+d_{3, f} & \forall f \\
w_{f}=\text { wor }_{f} x_{f} & \forall f \\
g_{f}=\operatorname{gor}_{f} x_{f} & \forall f
\end{array}
$$

The next section reviews several approaches to model and solve the development planning problem in the literature for the deterministic case where all the model parameters are assumed to be known with certainty. A generic MINLP model for oilfield development planning is presented next taking the infrastructure and reservoir characteristics presented in this section as reference. 


\section{Development Planning under Perfect Information}

The oilfield investment and operation planning has traditionally been modeled as LP (Lee and Aranofsky (1958), Aronofsky and Williams (1962)) or MILP (Frair, 1973) models under certain assumptions to make them computationally tractable. Simultaneous optimization of the investment and operation decisions was addressed in Bohannon (1970), Sullivan (1982) and Haugland et al. (1988) using MILP formulations with different levels of details in these models. Behrenbruch (1993) emphasized the need to consider a correct geological model and to incorporate flexibility into the decision process for an oilfield development project.

Iyer et al. (1998) proposed a multiperiod MILP model for optimal planning and scheduling of offshore oilfield infrastructure investment and operations. The model considers the facility allocation, production planning, and scheduling within a single model and incorporates the reservoir performance, surface pressure constraints, and oil rig resource constraints. To solve the resulting large-scale problem, the nonlinear reservoir performance equations are approximated through piecewise linear approximations. As the model considers the performance of each individual well, it becomes expensive to solve for realistic multi-field sites. Moreover, the flow rate of water was not considered explicitly for facility capacity calculations.

Van den Heever and Grossmann (2000) extended the work of Iyer et al. (1998) and proposed a multiperiod generalized disjunctive programming model for oil field infrastructure planning for which they developed a bilevel decomposition method. As opposed to Iyer and Grossmann (1998), they explicitly incorporated a nonlinear reservoir model into the formulation but did not consider the drill-rig limitations.

Grothey and McKinnon (2000) addressed an operational planning problem using an MINLP formulation where gas has to be injected into a network of low pressure oil wells to induce flow from these wells. Lagrangean decomposition and Benders decomposition algorithms were also proposed for the efficient solution of the model. Kosmidis et al. (2002) considered a production system for oil and gas consisting of a reservoir with several wells, headers and separators. The authors presented a mixed integer dynamic optimization model and an efficient approximation solution strategy for this system.

Barnes et al. (2002) optimized the production capacity of a platform and the drilling decisions for wells associated with this platform. The authors addressed this problem by solving a sequence of MILPs. Ortiz-Gomez et al. (2002) presented three mixed integer multiperiod 
optimization models of varying complexity for the oil production planning. The problem considers fixed topology and is concerned with the decisions involving the oil production profiles and operation/shut in times of the wells in each time period assuming nonlinear reservoir behavior.

Lin and Floudas (2003) considered the long-term investment and operations planning of the integrated gas field site. A continuous-time modeling and optimization approach was proposed introducing the concept of event points and allowing the well platforms to come online at potentially any time within planning horizon. Two-level solution framework was developed to solve the resulting MINLP problems which showed that the continuous time approach can reduce the computational efforts substantially and solve problems that were intractable for the discrete-time model.

Kosmidis et al. (2005) presented a mixed integer nonlinear (MINLP) model for the daily well scheduling in petroleum fields, where the nonlinear reservoir behaviour, the multiphase flow in wells and constraints from the surface facilities were simultaneously considered. The authors also proposed a solution strategy involving logic constraints, piecewise linear approximations of each well model and an outer approximation based algorithm. Results showed an increase in oil production up to $10 \%$ compared to a typical heuristic rules widely applied in practice.

Carvalho and Pinto (2006) considered an MILP formulation for oilfield planning based on the model developed by Tsarbopoulou (2000), and proposed a bilevel decomposition algorithm for solving large scale problems where the master problem determines the assignment of platforms to wells and a planning subproblem calculates the timing for the fixed assignments. The work was further extended by Carvalho and Pinto (2006) to consider multiple reservoirs within the model.

Barnes et al. (2007) addressed the optimal design and operational management of offshore oil fields where at the design stage the optimal production capacity of a main field was determined with an adjacent satellite field and a well drilling schedule. The problem was formulated as an MILP model. Continuous variables involved individual well, jacket and topsides costs, whereas binary variables were used to select individual wells within a defined field grid. An MINLP model wad proposed for the operational management to model the pressure drops in pipes and wells for multiphase flow. Non-linear cost equations were derived for the production costs of each well accounting for the length, the production rate and their maintenance. Operational 
decisions included the oil flowrates, the operation/shut-in for each well and the pressures for each point in the piping network.

Gunnerud and Foss (2010) considered the real-time optimization of oil production systems with a decentralized structure and modeled nonlinearities by piecewise linear approximations, resulting in a MILP model. The Lagrange relaxation and Dantzig-Wolfe decomposition methods were studied on a semi-realistic model of the Troll west oil rim which showed that both the approaches offers an interesting option to solve the complex oil production systems as compared to the fullspace method.

\section{(a) Key issues and Discussions}

The work described above uses a deterministic approach to address the oilfield development planning problem, and considers a sub-set of decisions under certain assumptions to ease the computational burden. One such approach used is to optimize the production profiles and other operation related decisions assuming that the investment decisions have already been fixed. Other simplifying approaches include optimizing the decisions associated with a field or a facility independent of decisions for other fields, optimizing investment and operation decisions assuming linear or piece-wise linear reservoir behavior, simplified reservoir characteristics, etc.

In this paper, we emphasize the need to include the following details and decisions in the deterministic model for it to be more realistic and consider various trade-offs to yield optimal investment and operations decisions in a multi-field setting:

1) All three components (oil, water and gas) should be considered explicitly in the formulation to consider realistic problems for facility installation and capacity decisions.

2) Nonlinear reservoir behavior in the model should be approximated by nonlinear functions such as higher order polynomials to ensure sufficient accuracy for the predicted reservoir profiles.

3) Reservoir profiles should be specific to the field-facility connections.

4) The number of wells should be a variable for each field to capture the realistic drill rig limitations and the resulting trade-offs among various fields.

5) The possibility of expanding the facility capacities in the future, and including the lead times for construction and expansions for each facility are essential to ensure realistic investments. 
6) Reservoir profiles should be expressed in such a way so that non-convexities can be minimized in the model for it to be computationally efficient.

7) The planning horizon should be long enough, typically 20-30 years.

Notice that the inclusion of the other details in the model could further improve the quality of decisions that are made. However, the model may become computationally intractable for the deterministic case itself. Therefore, it is assumed that accounting for the above details will provide a model that while being computationally tractable, is realistic.

\section{(b) Proposed Deterministic Development Planning Model}

We outline in this section the proposed MINLP model (Gupta and Grossmann, 2011), in which we incorporate all the features mentioned above. The model takes the infrastructure (Fig. 4) and reservoir characteristics (Eq. (a)-(f)) presented in the earlier section as reference. With the objective of maximizing the NPV considering a long-term planning horizon, the key investment and operations decisions included in the proposed multi-period planning model are as follows:

(i) Investment decisions in each time period include which FPSO facilities should be installed or expanded, and their respective installation or expansion capacities for oil, liquid and gas, which fields should be connected to which FPSO facility, and the number of wells that should be drilled in a particular field given the restrictions on the total number of wells that can be drilled in each time period over all the given fields.

(ii) Operating decisions include the oil/gas production rates from each field in each time period.

It is assumed that all the installation and expansion decisions occur at the beginning of each time period, while operation takes place throughout the time period at constant conditions. There is a limit on the number of expansions for each FPSO facility, and lead time for its initial installation and expansion decision. The above decisions should satisfy the following set of constraints:

Economic Constraints: The gross revenues, based on the total amount of oil and gas produced, and total cost based on capital and operating expenses in each time period are calculated in these constraints. Capital costs consist of the fixed FPSO installation cost, variable installation and expansion costs, field-FPSO connection costs and well drilling costs in each time period, while total operating expenses depend on the total amount of liquid and gas produced. 
Reservoir Constraints: These constraints predict the reservoir production performance for each field in each time period. In particular, the oil flow rate from each well for a particular FPSOfield connection to be less than the deliverability (maximum oil flow rate) of that field. The cumulative water and cumulative gas produced by the end of time period from a field, are represented by a polynomial in terms of fractional oil recovery by the end of time period, and are further used to calculate individual water and gas flowrates. The cumulative oil produced is also restricted by the recoverable amount of oil from the field. The other way to incorporate water and gas flow rates is to use the water-oil-ratio and gas-oil-ratio profiles directly in the model, eqs. (c)-(d). However, it will add bilinear terms in the model, eqs. (e)-(f).

Field-FPSO Flow constraints: It includes the material balance constraints for the flow between fields and FPSOs. In particular, the total oil flow rate from field in time period is the sum of the oil flow rates over all FPSO facilities from this field, which depends on the oil flow rate per well and number of wells available for production. Total oil, water and gas flowrates into each FPSO facility, at time period from all the given fields, is calculated as the sum of the flow rates of each component over all the connected fields.

FPSO Capacity Constraints: These equations restrict the total oil, liquid and gas flow rates into each FPSO facility to be less than its corresponding capacity in each time period. The FPSO facility capacities in each time period are computed as the sum of the corresponding installation and expansion capacities taking lead times into considerations. Furthermore, there are restrictions on the maximum installation and expansion capacities for each FPSO facility.

Well drilling limitations: The number of wells available in a field for drilling is calculated as the sum of the wells available at the end of the previous time period and the number of wells drilled at the beginning of time period. The maximum number of wells that can be drilled over all the fields during each time period and in each field during complete planning horizon, are restricted by the respective upper bounds.

Logic Constraints: Logic constraints include the restrictions on the number of installation and expansion of a FPSO facility, and possible FPSO-field connections during the planning horizon. Other logic constraints are also included to ensure that the FPSO facility can be expanded, and the connection between a field and that facility and corresponding flow can occur only if that facility has already been installed by that time period. 
The proposed non-convex MINLP model for offshore oilfield planning involves nonlinear and non-convex constraints that can lead to suboptimal solutions when solved with a method that assumes convexity (e.g. branch and bound, outer-approximation). The detailed description of the model is outlined in Gupta and Grossmann (2011) with two possibilities of MINLP formulations. MINLP Model 1 consists of bilinear terms in the formulation involving WOR, GOR and oil flow rates. MINLP model 2 includes univariate polynomials that represent reservoir profiles in terms of cumulative water and gas produced. In addition, some constraints that involves bilinear terms with integer variables that calculates the total oil flow rate from a field as the multiplication of the number of available wells in the field and oil flow rate per well, are also present. However, this MINLP formulation (Model 2) can be reformulated into an MILP using piecewise linearizations and exact linearizations with which the problem can be solved to global optimality (Gupta and Grossmann, 2011).

Table 1 summarizes the main features of these MINLP and reformulated MILP models. In particular, the reservoir profiles and respective nonlinearities involved in the models are compared in the table. Realistic instances involving 10 fields, 3 FPSO's and 20 years planning horizon have been solved and comparisons of the computational performance of the proposed MINLP and MILP formulations are presented in the paper. The computational efficiency of the proposed MINLP and MILP models have been further improved by binary reduction scheme that yield an order of magnitude reduction in the solution time. A large scale example is explained in the results section 6 of this paper.

Table 1: Comparison of the nonlinearities involved in 3 model types

\begin{tabular}{|c|c|c|c|}
\hline & Model 1 & Model 2 & Model 3 \\
\hline Model Type & MINLP & MINLP & MILP \\
\hline Oil Deliverability & $3^{\text {rd }}$ order polynomial & $3^{\text {rd }}$ order polynomial & Piecewise Linear \\
\hline WOR & $3^{\text {rd }}$ order polynomial & - & - \\
\hline GOR & $3^{\text {rd }}$ order polynomial & - & - \\
\hline wc & - & $4^{\text {th }}$ order polynomial & Piecewise Linear \\
\hline gc & - & $4^{\text {th }}$ order polynomial & Piecewise Linear \\
\hline Bilinear Terms & $\begin{array}{c}\mathrm{N} * \mathrm{x} \\
\mathrm{N} \mathrm{x}^{*} \mathrm{WOR} \\
\mathrm{N}^{*} \mathrm{x} * \mathrm{GOR}\end{array}$ & $\mathrm{N}^{*} \mathrm{x}$ & None \\
\hline MILP Reformulation & Not Possible & Possible & Reformulated MILP \\
\hline
\end{tabular}




\section{Remarks:}

The proposed non-convex MINLP model yields good quality solutions in few seconds when solving with DICOPT directly even for large instances. There are various trade-offs involve in selecting a particular model for oilfield problem. In case that we are concerned with the solution time, especially for the large instances, it would be better to use DICOPT on the MINLP formulations directly to obtain good quality solutions with modest computational times, although global optimality is not guaranteed. If computing times are of no concern, one may want to use the MILP approximation models that can yield better solutions, but at a higher computational cost as explained in the results section.

Furthermore, these MILP solutions also provide a way to assess the quality of the suboptimal solutions from the MINLPs, or finding better once using its solution for the original problem. These MINLP or MILP models can further be used as the basis to exploit various decomposition strategies or global optimization techniques for solving the problems to global optimality. Moreover, the deterministic model proposed in the paper is very generic and can either be used for simplified cases (e.g. linear profiles for reservoir, fixed well schedule, single field site etc.), or extended to include other complexities as discussed in the following sections.

\section{Incorporating Uncertainty in the Development Planning}

In the previous section, one of the major assumptions is that there is no uncertainty in the model parameters, which in practice is generally not true. There are multiple sources of uncertainty in these projects. The market price of oil/gas, quantity and quality of reserves at a field are the most important sources of the uncertainty in this context. The uncertainty in oil prices is influenced by the political, economic or other market factors. The uncertainty in the reserves on the other hand, is linked to the accuracy of the reservoir data (technical uncertainty). While the existence of oil and gas at a field is indicated by seismic surveys and preliminary exploratory tests, the actual amount of oil in a field, and the efficacy of extracting the oil will only be known after capital investment have been made at the field. Both, the price of oil and the quality of reserves directly affect the overall profitability of a project, and hence it is important to consider the impact of these uncertainties when formulating the decision policy. However, the problem that addresses the issue of uncertainty within development planning is very challenging due to the additional complexity caused by the model size and resulting increase in the solution time. 
Although limited, there has been some work that accounts for uncertainty in the problem of optimal development of oil and/or gas fields. Haugen (1996) proposed a single parameter representation for uncertainty in the size of reserves and incorporates it into a stochastic dynamic programming model for scheduling of oil fields. However, only decisions related to the scheduling of fields were considered. Meister et al. (1996) presented a model to derive exploration and production strategies for one field under uncertainty in reserves and future oil prices. The model was analyzed using stochastic control techniques.

Jonsbraten (1998) addressed the oilfield development planning problem under oil price uncertainty using an MILP formulation that was solved with a progressive hedging algorithm. Aseeri et al. (2004) introduced uncertainty in the oil prices and well productivity indexes, financial risk management, and budgeting constraints into the model proposed by Iyer and Grossmann (1998), and solved the resulting stochastic model using a sampling average approximation algorithm.

Jonsbraten (1998b) presented an implicit enumeration algorithm for the sequencing of oil wells under uncertainty in the size and quality of oil reserves. The author uses a Bayesian approach to represent the resolution of uncertainty with investments. Both these papers consider investment and operation decisions for one field only. Lund (2000) addressed a stochastic dynamic programming model for evaluating the value of flexibility in offshore development projects under uncertainty in future oil prices and in the reserves of one field using simplified descriptions of the main variables.

Cullick et al. (2003) proposed a model based on the integration of a global optimization search algorithm, a finite-difference reservoir simulation, and economics. In the solution algorithm, new decision variables were generated using meta-heuristics, and uncertainties were handled through simulations for fixed design variables. They presented examples having multiple oil fields with uncertainties in the reservoir volume, fluid quality, deliverability, and costs. Few other papers, (Begg et al. (2001), Zabalza-Mezghani et al. (2004), Bailey et al. (2005), Cullick et al. (2007)), have also used a combination of reservoir modeling, economics and decision making under uncertainty through simulation-optimization frameworks.

Ulstein et al. (2007) addressed the tactical planning of petroleum production that involves regulation of production levels from wells, splitting of production flows into oil and gas 
products, further processing of gas and transportation in a pipeline network. The model was solved for different cases with demand variations, quality constraints, and system breakdowns.

Elgsæter et al. (2010) proposed a structured approach to optimize offshore oil and gas production with uncertain models that iteratively updates setpoints, while documenting the benefits of each proposed setpoint change through excitation planning and result analysis. The approach is able to realize a significant portion of the available profit potential, while ensuring feasibility despite large initial model uncertainty.

However, most of these works either consider the very limited flexibility in the investment and operations decisions or handle the uncertainty in an ad-hoc manner. Stochastic programming provides a systematic framework to model problems that require decision-making in the presence of uncertainty by taking uncertainty into account of one or more parameters in terms of probability distribution functions, (Birge and Louveaux, 1997). This area has been receiving increasing attention given the limitations of deterministic models. The concept of recourse action in the future, and availability of probability distribution in the context of oilfield development planning problems, makes it one of the most suitable candidates to address uncertainty. Moreover, extremely conservative decisions are usually ignored in the solution utilizing the

probability information given the potential of high expected profits in the case of favorable outcomes.

In the next section, we first provide a basic background on the stochastic programming. Furtheremore, given the importnace of uncertianty in the reserves sizes and its quality (decisiondependent uncertianty) that directly impact the profitabiltiy of the project, a detailed review of the model and solution methods recently proposed are discussed (Goel and Grossmann (2004), Goel et al. (2006), Tarhan et al. (2009)).

\section{(a) Basics Elements of Stochastic Programming}

A Stochastic Program is a mathematical program in which some of the parameters defining a problem instance are random (e.g. demand, yield). The basic idea behind stochastic programming is to make some decisions now (stage 1) and to take some corrective action (recourse) in the future, after revelation of the uncertainty (stages $2,3, \ldots$ ). If there are only two stages, then the problem corresponds to a 2-stage stochastic program, while in a multistage stochastic program the uncertainty is revealed sequentially, i.e. in multiple stages (time periods), 
and the decision-maker can take corrective action over a sequence of stages. In the two-stage and multistage case the cost of the decisions and the expected cost of the recourse actions are optimized.

The problems are usually formulated under the assumption that uncertain parameters follow discrete probability distributions, and that the planning horizon consists of a fixed number of time periods that correspond to decision points. Using these two assumptions, the stochastic process can be represented with scenario trees. In a scenario tree (Figure 6-a) each node represents a possible state of the system at a given time period. Each arc represents the possible transition from one state in time period $t$ to another state in time period $t+1$, where each state is associated with the probabilistic outcome of a given uncertain parameter. A path from the root node to a leaf node represents a scenario.
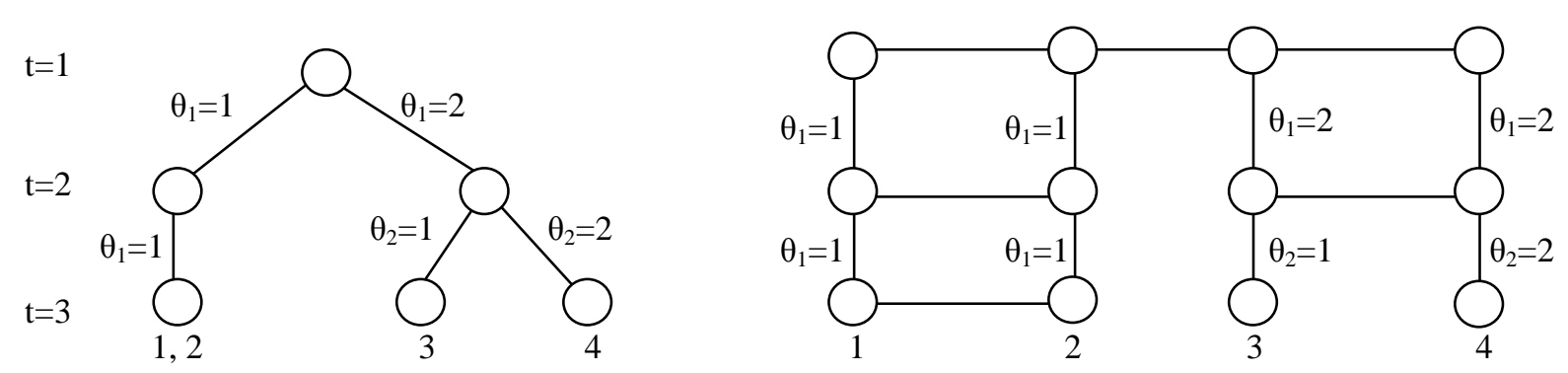

(a) Standard Scenario Tree with uncertain parameters $\theta_{1}$ and $\theta_{2}$

(b) Alternative Scenario Tree

Figure 6: Tree representations for discrete uncertainties over 3 stages.

An alternative representation of the scenario tree was proposed by Ruszczynski (1997) where each scenario is represented by a set of unique nodes (Figure 6-b). The horizontal lines connecting nodes in time period $t$, mean that nodes are identical as they have the same information, and those scenarios are said to be indistinguishable in that time period. These horizontal lines correspond to the non-anticipativity (NA) constraints in the model that link different scenarios and prevent the problem from being decomposable. The alternative scenario tree representation allows to model the uncertainty in the problem more effectively.

Jonsbraten (1998) classified uncertainty in Stochastic Programming problems into two broad categories: exogenous uncertainty where stochastic processes are independent of decisions that are taken (e.g. demands, prices), and endogenous uncertainty where stochastic processes are affected by these decisions (e.g. reservoir size and its quality). Notice that the resulting scenario tree in the exogenous case is decision independent and fixed, whereas endogenous uncertainty 
problems yield a decision-dependent scenario tree. In the process systems area, Ierapetritou and Pistikopoulos (1994), Clay and Grossmann (1997) and Iyer and Grossmann (1998) solved various production planning problems that considered exogenous uncertainty and formulated as the two-stage stochastic programs. Furthermore, detailed reviews of previous work on problems with exogenous uncertainty can be found in Schultz (2003) and Sahinidis (2004). These approaches for exogenous uncertainty can directly be exploited for the oilfield development planning problem under oil/gas price uncertainty. In this paper, we focus on the endogenous uncertainty problems where limited literature is available.

In the next section, we review the development planning problem using a multistage stochastic programming (MSSP) approach with endogenous uncertainty, where the structure of scenario tree is decision-dependent.

\section{(b) Development planning under Endogenous Uncertainty}

Most previous work in planning under uncertainty has considered exogenous uncertainty where stochastic processes are independent of decisions (e.g., demands, prices). In contrast, there is very limited work in problems in which the stochastic processes are affected by decisions, that is with endogenous uncertainty.

Decisions can affect stochastic processes in two different ways (Goel and Grossmann, 2006). Either they can alter the probability distributions (type 1), or they can be used to discover more accurate information (type 2). In this paper, we focus on the type 2 of endogenous uncertainty where the decisions are used to gain more information, and eventually resolve uncertainty.

Ahmed (2000), Vishwanath et al. (2004) and Held et al. (2005) considers type 1 decisiondependent uncertainty. Ahmed (2000) presented examples on network design, server selection, and facility location problems with decision-dependent uncertainties that are reformulated as MILP problems, and solved by LP-based branch-and-bound algorithms. Vishwanath et al. (2004) addressed a network problem having endogenous uncertainty in survival distributions. The problem is a two-stage stochastic program in which first-period investment decisions are made for changing the survival probability distribution of arcs after a disaster. The aim is to find the investments that minimizes the expected shortest path from source to destination after a disaster. Held et al. (2005) considered the problem that includes endogenous uncertainty in the structure

of a network. In each stage of this problem, an operator tries to find the shortest path from a 
source to a destination after some of the nodes in the network are blocked. The aim is to maximize the probability of stopping the flow of goods or information in the network.

Another way the decisions can impact the stochastic process is that they can affect the resolution of uncertainty or the time uncertainty resolves (type 2). Type 2 uncertainty can further be classified into two categories.

\section{Immediate Uncertainty Resolution:}

The first category of type 2 decision-dependent uncertainty assumes that the revelation of accurate information (resolution of endogenous uncertainty) occurs instantaneously (Pflug (1990); Jonsbraten et al. (1998); Goel and Grossmann (2004, 2006); Goel et al. (2006); Boland et al. (2008)).

Pflug (1990) addressed endogenous uncertainty problems in the context of discrete event dynamic systems where the underlying stochastic process depends on the optimization decisions. Jonsbraten et al. (1998) proposed an implicit enumeration algorithm for the problems in this class where decisions that affect the uncertain parameter values are made at the first stage.

In the context of oilfield development planning, Goel and Grossmann (2004) considered a gas field development problem under uncertainty in the size and quality of reserves where decisions on the timing of field drilling were assumed to yield an immediate resolution of the uncertainty, i.e. the problem involves decision-dependent uncertainty as discussed in Jonsbraten et al. (1998). The alternative scenario tree representation described earlier is used as a key element to model the problem in this work. Linear reservoir models, which can provide a reasonable approximation for gas fields, were used. In their solution strategy, the authors used a relaxation problem to predict upper bounds, and solved multistage stochastic programs for a fixed scenario tree for finding lower bounds. Goel et al. (2006) later proposed the theoretical conditions to reduce the number of non-anticipativity constraints in the model. The authors also developed a branch and bound algorithm for solving the corresponding disjunctive/mixed-integer programming model where lower bounds are generated by Lagrangean duality. The proposed decomposition strategy relies on relaxing the disjunctions and logic constraints for the nonanticipativity constraints.

Boland et al. (2008) applied multistage stochastic programming to open pit mine production scheduling, which is modeled as a mixed-integer linear program. These authors consider the 
endogenous uncertainty in the total amount of rock and metal contained in it, where the excavation decisions resolve this uncertainty immediately. They followed a similar approach as Goel and Grossmann (2006) for modeling the problem, with the exception of eliminating some of the binary variables used in the general formulation to represent conditional nonanticipativity constraints. Furthermore, they solved the model in full-space without using a decomposition algorithm. These authors also compared the fullspace results for this mine-scheduling problem with the one where non-anticipativity constraints were treated as 'lazy constraints' during the solution in CPLEX.

Colvin and Maravelias $(2008,2010)$ presented several theoretical properties, specifically for the problem of scheduling of clinical trials having uncertain outcomes in the pharmaceutical R\&D pipeline. These authors developed a branch and cut framework to solve these MSSP problems with endogenous uncertainty under the assumption that only few non-anticipativity constraints be active at the optimal solution.

Gupta and Grossmann (2011) proposed a generic mixed-integer linear multistage stochastic programming model for the problems with endogenous uncertainty where uncertainty in the parameters resolve immediately based on the investment decisions. The authors exploit the problem structure and extend the conditions by Goel and Grossmann (2006) to formulate a reduced model to improve the computational efficiency in fullspace. Furthermore, several generic solution strategies for the problems in this class are proposed to solve the large instances of these problems, with numerical results on process networks examples.

Ettehad et al. (2011) presented a case study for the development planning of an offshore gas

field under uncertainty optimizing facility size, well counts, compression power and production policy. A two-stage stochastic programming model was developed to investigate the impact of uncertainties in original gas in place and inter-compartment transmissibility. Results of two solution methods, optimization with Monte Carlo sampling and stochastic programming, were compared which showed that the stochastic programming approach is more efficient. The models were also used in a value of information (VOI) analysis.

\section{Gradual uncertainty Resolution:}

The second category of decision-dependent uncertainty of type 2 assumes that uncertainty resolves gradually over time because of learning (Tarhan and Grossmann (2008); Solak (2007); 
Tarhan et al. (2009); Stensland and Tjøstheim (1991); Dias (2002); Jonsbraten (1998); Harrison (2007)).

Stensland and Tjøstheim (1991) have worked on a discrete time problem for finding optimal decisions with uncertainty reduction over time and applied their approach to oil production. These authors expressed the uncertainty in terms of a number of production scenarios. Their main contribution was combining production scenarios and uncertainty reduction effectively for making optimal decisions. Dias (2002) presented four propositions to characterize technical uncertainty and the concept of revelation towards the true value of the variable. These four propositions, based on the theory of conditional expectations, are employed to model technical uncertainty.

Jonsbraten (1998) considered gradual uncertainty reduction where all uncertainty is assumed to resolve at the end of the project horizon. The author used a decision tree approach for modeling the problem where Bayesian statistics are applied to find the probabilities of branches in the decision tree that are decision dependent. The author also proposed an algorithm that relies on the prediction of upper and lower bounds. Harrison (2007) used a different approach for optimizing two-stage decision making problems under uncertainty. Some of the uncertainty was assumed to resolve after the observation of the outcome of the first stage decision. The author developed a new method, called Bayesian Programming, where the corresponding integrals were approximated using Markov Chain Monte Carlo simulations, and decisions were optimized using simulated annealing type of meta-heuristics.

Solak (2007) considered the project portfolio optimization problem that deals with the selection of research and development projects and determination of optimal resource allocations under decision dependent uncertainty where uncertainty is resolved gradually. The author used the sample average approximation method for solving the problem, where the sample problems were solved through Lagrangean relaxation and heuristics.

Tarhan and Grossmann (2008) considered the synthesis of process networks with uncertainties in the yields of the processes, which are resolved gradually over time depending on the investment and operating decisions. In the context of oilfield development planning problem, Tarhan et al. (2009) address the planning of offshore oil field infrastructure involving endogenous uncertainty in the initial maximum oil flowrate, recoverable oil volume, and water breakthrough time of the reservoir, where decisions affect the resolution of these uncertainties. 
The authors extend the work of Goel and Grossmann (2004) and Goel et al. (2006) but with three major differences.

i. Model focuses on a single field consisting of several reservoirs rather than multiple fields. However, more detailed decisions such as the number, type, and construction decisions for infrastructure are considered.

ii. Nonlinear, rather than linear, reservoir models are considered. Nonlinear reservoir models are important because Goel and Grossmann (2004) focused on gas fields, for which linear models are often an adequate approximation, while for oil fields nonlinear reservoir models are required.

iii. The resolution of uncertainty is gradual over time instead of being resolved immediately. Compared to the instantaneous uncertainty resolution, gradual resolution gives rise to some challenges in the model, including the underlying scenario tree and the nonanticipativity constraints.

Tarhan et al. (2009) developed a multistage stochastic programming framework that was modeled as a disjunctive/mixed-integer nonlinear programming model consisting of individual non-convex MINLP subproblems connected to each other through initial and conditional nonanticipativity constraints. A duality-based branch and bound algorithm was proposed taking advantage of the problem structure and globally optimizing each scenario problem independently. An improved solution approach was also proposed that combines global optimization and outer-approximation to optimize the investment and operations decisions (Tarhan et al. (2011)).

\section{(c) Discussions}

The explicit modeling of endogenous uncertainty in the multistage stochastic programming framework, and the proposed duality based branch and bound solution strategies (Goel et al. 2006, Tarhan et al. 2009, 2011) can be very useful for the oilfield development planning problem. The examples considered in these papers show considerable improvement in the expected NPV compared to the solution where uncertainty is handled by simply using expected values of the parameters (expected value solution). The added value of stochastic programming is due to the more conservative initial investment strategy compared to the expected value solution. In particular, stochastic programming solution proposes higher investments only when 
the uncertainty parameters are found to be favorable. Moreover, stochastic programming based model generate solutions that not only provides higher expected net present values, but also offers more robust solutions with respect to the uncertainties that are not included explicitly in the uncertainty space. The stochastic solutions have low risk in terms of probability of leading to a negative NPV as compared to expected value solution. These results support the advantage of using stochastic programming approach for development planning of oilfields.

The model considered by Goel et al. (2006) is for gas fields only, where linear reservoir profiles is a reasonable assumption. The model considers multiple fields but facility expansions and well drilling decisions are not considered. On the other hand, the model considered in Tarhan et al. (2009) assumes either gas/water or oil/water components for a single field and single reservoir at a detailed level. Hence, its extension to realistic multiple field instances can be expensive to solve with this model. Also, the model cannot be reformulated as an MILP for solving large instances to global optimality.

Therefore, the proposed deterministic model, (Gupta and Grossmann, 2011), presented in the earlier section can be used as a basis to incorporate uncertainty in the decision-making process (either exogenous or endogenous) under the proposed unified framework (Fig. 1). The model includes multiple fields, oil, water and gas, nonlinear reservoir behavior, well drilling schedule and facility expansions and lead times to represent realistic oilfield development project while being computationally tractable for large instances. The interaction among various fields, possibility of capacity expansions, lead-times and well drilling decisions allows incorporating recourse actions in a more practical way. Moreover, the model is suitable for either immediate (Goel et al. 2006) or gradual uncertainty resolution (Tarhan et al. 2009) using multistage stochastic program as the underlying model.

However, there are still certain points in the proposed uncertainty modeling approaches and solution algorithms in these papers (Goel et al. 2006, Tarhan et al. 2009) for which there is scope for further improvement:

1. The duality based branch and bound method can further be improved e.g. use of logic inference during the generation of cuts in the branch and bound tree, robust procedure to generate feasible solution from the solution of Lagrangean dual, use of parallel computing and scenario reduction algorithms to solve large instances etc. 
2. Other solution approaches (e.g. Gupta and Grossmann (2011)) for multiage stochastic problems with endogenous uncertainties can be explored.

3. Some heuristic approaches and model approximations can be explored to handle the realistic large scale development planning problems with uncertainty.

4. The importance of considering other uncertain parameters, most notably oil price, might be interesting to consider.

5. The decision maker is assumed to be risk neutral in the proposed models where the objective is to maximize the expected net present value. In reality with such huge investments, companies are interested in models that consider not only uncertainty but also the risk explicitly, which can make the problem even more challenging to solve (You et al. 2009).

6. The available reservoir simulators ECLIPSE (Schlumberger, 2008) could be incorporated into the model to improve the accuracy of the reservoir profiles, although the potential computational expense would be too large.

7. Although the real options methods, (Lund (1999, 2005), Kalligeros (2004), Dias (2004)), seems to be limited to the number of decisions and the flexibility to incorporate complex system structures, some insight about its advantages and disadvantages over stochastic programming methods would be worth investigating.

It should also be noted that the development planning models are not intended to solve only once to plan for next 20-30 years. Instead the model can be updated and resolved multiple times as more information reveals.

\section{Development Planning with Fiscal Considerations}

In previous sections, we considered the development planning problem under perfect information and proposed a deterministic model that can be applied to a project with multiple fields. Furthermore, we also outlined the multistage stochastic approaches to incorporate uncertainty and their solution methods. The deterministic model, (Gupta and Grossmann, 2011), can be used a basis to incorporate uncertainty according to the unified framework (Fig. 1).

Including fiscal considerations, (Van den Heever and Grossmann (2001), Lin and Floudas (2003)), as part of the investment and operation decisions for the oilfield development problem can significantly impact the optimal investment and operations decisions and actual NPV. 
Therefore, in this section we extend the proposed deterministic model that considers multiple oil and gas fields with sufficient detail to include generic complex fiscal rules in development planning under the proposed framework (Fig. 1). We first consider the basic elements of the various types of contracts involved in this industry, review the work in this area and provide a generic approach to include these contracts terms in the model.

\section{(a) Type of Contracts}

There are a variety of contracts that are used in the offshore oil and gas industry (World Bank, 2007). These contracts can be classified into two main categories:

I. Concessionary System: A concessionary (or tax and royalty) system usually involves royalty, deduction and tax. Royalty is paid to the government at a certain percentage of the gross revenues each year. The net revenue after deducting costs becomes taxable income on which a pre-defined percentage is paid as tax. The total contractor's share involves gross revenues minus royalty and taxes. The basic difference as compared to the production sharing agreement, is that the oil company obtains the title to all of the oil and gas at the wellhead and pay royalties, bonuses, and other taxes to the government.

II. Production Sharing Agreements: The revenue flow in a typical Production Sharing Agreement can be seen as in Figure 7. Some portion of the total oil produced is kept as cost oil by the oil company for cost recovery purposes after paying royalties to the government that is a certain percentage of the oil produced. There is a cost recovery ceiling to ensure revenues to the government as soon as production starts. The remaining part of the oil called profit oil is divided between oil company and the host government. The oil company needs to further pay income tax on its share of profit oil. Hence, the total contractor's (oil company) share in the gross revenue comprises of cost oil and contractor's profit oil share after tax. The other important feature of a PSA is that the government owns all the oil and transfers title to a portion of the extracted oil and gas to the contractor at an agreed delivery point. Notice that the cost oil limit is one of the key differences with a concessionary system. 


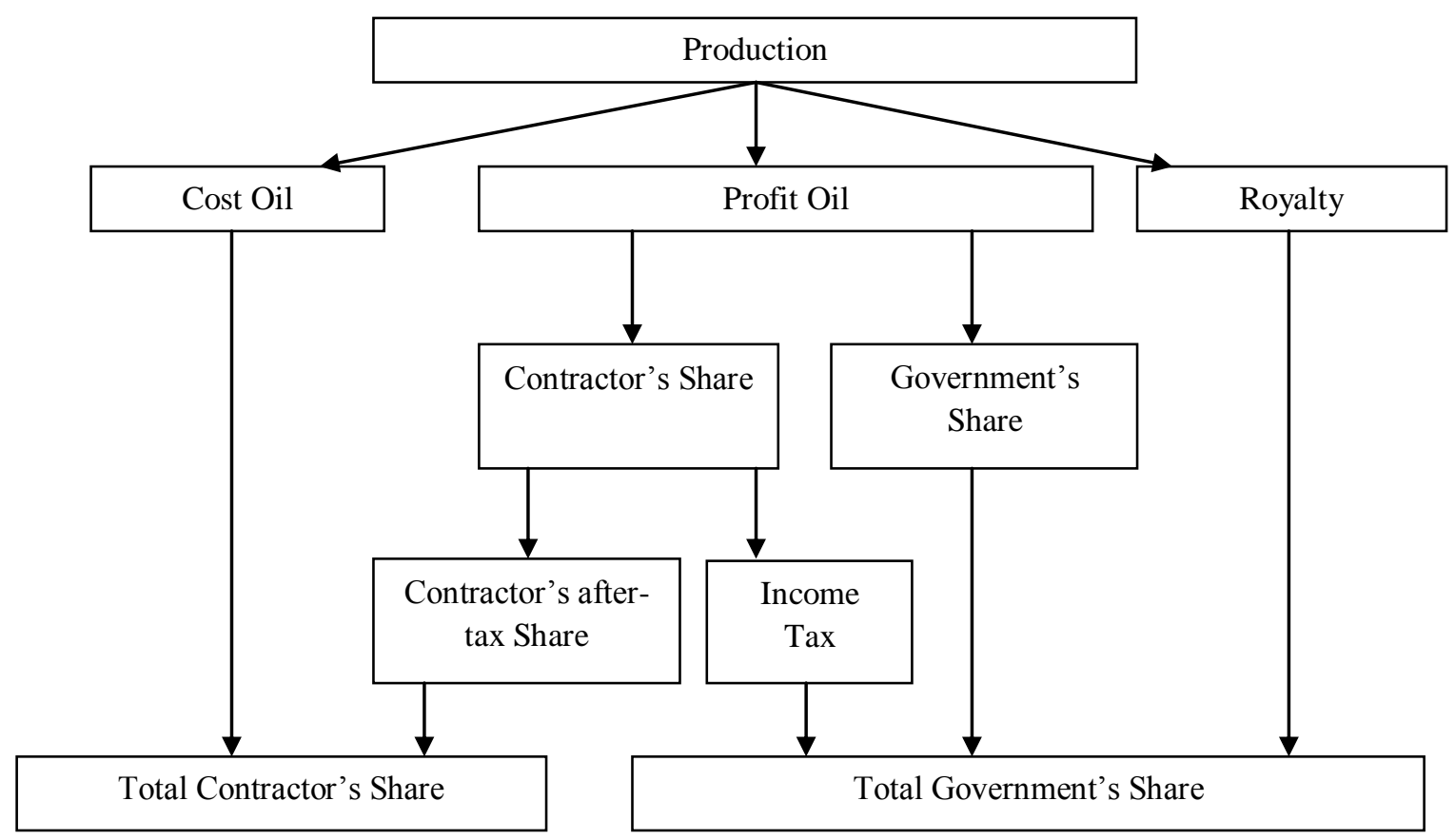

Figure 7: Revenue flow for a typical Production Sharing Agreement

The specific rules defined in such a contract (either PSA or concessionary) between operating oil company and host government determine the profit that the oil company can keep as well as the royalties and profit share that are paid to the government. These profit oil splits, royalty rates are usually based on the profitability of the project (progressive fiscal terms), where cumulative oil produced, rate of return, R-factor etc. are the typical profitability measures that determine the tier structure for these contract terms.

In particular, the fraction of total oil production to be paid to the government in terms of profit share, royalties are to be calculated based on the value of one or more profitability parameters (e.g. cumulative production, daily production, IRR, etc.), specifically in the case of progressive fiscal terms. The transition to the higher profit share, royalty rates is expressed in terms of tiers that are a step function ( $\mathrm{g}$ ) linked to the above parameters and corresponding threshold values. For instance, if the cumulative production is in the range of first tier, $L_{1} \leq x c_{t} \leq U_{1}$, the royalty $R_{l}$ will be paid to the government, while if the cumulative production reaches in tier 2 , royalty $R_{2}$ will need to be paid, and so on. In practice, as we move to the higher tier the percentage share of government in the total production increases. Notice that if the fiscal 
terms are not linked to the specific parameters, e.g. royalties, profit share are the fixed percentage of total production, then the tier structure is not present in the problem.

$$
\text { Royalty Rate }=\left\{\begin{array}{lll}
R_{1} & L_{1} \leq x c \leq U_{1} & \text { :Tier } 1 \\
R_{2} & L_{2} \leq x c \leq U_{3} & \text { :Tier } 2 \\
R_{3} & L_{3} \leq x c \leq U_{3} & \text { :Tier } 3 \\
R_{3} & L_{4} \leq x c \leq U_{4} & \text { :Tier } 4
\end{array}\right.
$$

Given that the resulting royalties and/or government profit oil share can be a significant amount of the gross revenues, it is critical to consider these contracts terms explicitly during oilfield planning to access the actual economic potential of such a project. For instance, a very promising oilfield or block can turn out to be a big loss or less profitable than projected in the long-term if significant royalties are attached to that field, which was not considered during the development planning phase involving large investments. On the contrary, there could be the possibility of missing an opportunity to invest in a field that has very difficult conditions for production and looks unattractive, but can have favorable fiscal terms resulting in large profits in the long-term. In the next section we discuss how to include these rules within a development planning model.

\section{(b) Development Planning Model with Fiscal Considerations}

The models and solutions approaches in the literature that consider the fiscal rules within development planning are either very specific or simplified, e.g. one field at a time, only one component, simulation or meta-heuristic based approaches to study the impact of fiscal terms that may not yield the optimal solutions (Sunley et al. (2002), Kaiser, M.J. and A.G. Pulsipher (2004), World Bank (2007), Tordo (2007)).

Van den Heever et al. (2000), and Van den Heever and Grossmann (2001) used a deterministic model to handle complex economic objectives including royalties, tariffs, and taxes for the multiple gas field site. These authors incorporated these complexities into their model through disjunctions as well as big-M formulations. The results were presented for realistic instances involving 16 fields and 15 years. However, the model considers only gas production and the number of wells were used as parameters (fixed well schedule) in the model. Moreover, the fiscal rules presented were specific to the gas field site considered for the study, but not in the generic form. Based on the continuous time formulation for gas field development with complex 
economics of similar nature as Van den Heever and Grossmann (2001), Lin and Floudas (2003) presented an MINLP model and solved it with a two stage algorithm.

With the motivation for optimal investment and operations decisions in a realistic situation for offshore oil and gas field planning project, we incorporate the generic fiscal terms described earlier within proposed planning models (MINLP and MILP) and unified framework (Fig. 1). Notice that we focus on the progressive production sharing agreement terms here that covers the key elements of the most of the available contracts, and represent one of the most generic forms of fiscal rules. Particular fiscal rules of interest can be modeled as the specific case of this representation.

The objective here is to maximize the contractor's NPV which is the difference between total contractor's revenue share and total cost occurred over the planning horizon, taking discounting into consideration. The idea of cost recovery ceiling is included in terms of min function (h) to limit the amount of total oil produced each year that can be used to recover the capital and operational expenses. This ceiling on the cost oil recovery is usually enforced to ensure early revenues to the Govt. as soon as production starts.

$$
C O_{t}=\min \left(C R_{t}, f_{t}^{C R} \cdot R E V_{t}\right) \quad \forall t
$$

Moreover, a sliding scale based profit oil share of contractor that is linked to some parameter, for instance cumulative oil production, is also included in the model. In particular, disjunction (i) is used to model this tier structure for profit oil split which states that variable $Z_{i, t}$ will be true if cumulative oil production by the end of time period $t$, (Gupta and Grossmann, 2012), lies between given tier thresholds $L_{i} \leq x c_{t} \leq U_{i}$, i.e. tier $i$ is active and split fraction $f_{i}^{P O}$ is used to determine the contractor share in that time period. The disjunction (i) in the model is further reformulated into linear and mixed-integer linear constraints using the convex-hull formulation (Lee and Grossmann, 2000).

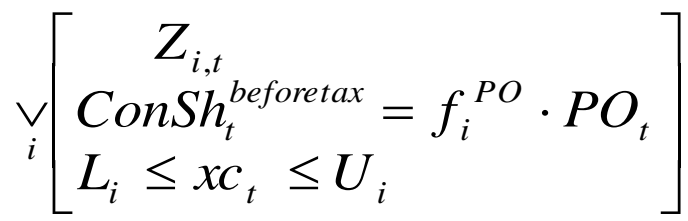

We assume that only profit oil split is based on a sliding scale system, while other fractions (e.g. tax rate, cost recovery limit fraction) are fixed parameters. Furthermore, the proposed model 
is also extended to include the ring-fencing, which is the provision that are usually part of fiscal terms and have significant impact on the NPV calculations. These provisions determine that all the costs associated with a given block (which may be a single field or a group of fields) or license must be recovered from revenues generated within that block, i.e. the block is "ringfenced". It basically defines the level at which all fiscal calculations are need to be done, and provide restriction to balance the costs and revenues across various projects/blocks that are not part of that ring-fence. The other constraints and features remains the same as the proposed MINLP and MILP models described in earlier sections.

Notice that the deterministic model with fiscal consideration presented here can also be used as the basis for the stochastic programming approaches explained in the previous section to incorporate uncertainty in the model under the unified framework (Fig. 1). Optimal investment and operations decisions, and the computational impact of adding a typical progressive Production Sharing Agreement (PSA) terms, is demonstrated in the results section 6 with a small example.

\section{(c) Key issues and Discussions}

The extension of the deterministic oilfield development planning problem to include fiscal rules explicitly in the formulation raises the following issues:

1. The model can become expensive to solve with the fiscal rules, especially the one that involves progressive fiscal terms due to the additional binary variable that are required to model the tier structure and resulting weak relaxation of the model. This is due to the relatively weak bounds on some of the key variables in the model, e.g. contractor share, profit oil, cost oil etc. which are difficult to estimate a priori.

2. Specialized decomposition algorithms that exploit the problem structure may be exploited to improve the computational efficiency.

3. Alternative ways to disjunctive models for the fiscal rules can be analyzed, e.g. including fiscal terms in approximate form, especially for the progressive fiscal terms so that multifield site problem is tractable.

4. Although we include the most general basic elements of the fiscal rules, there might be some additional project specific fiscal terms that can have significant impact. Therefore, it is important to include the corresponding fiscal terms defined for a particular project of interest. 
In a forthcoming paper, we will discuss the details of the generic model for the development planning problem with fiscal considerations and ways to improve its computational efficiency, (Gupta and Grossmann, 2012).

\section{Examples}

In this section we consider a variety of the examples for the oilfield development planning problem that covers deterministic, stochastic and complex fiscal features as discussed in the earlier sections.

\section{(a) Instance 1: Deterministic Case}

In this section we present an example of the oilfield planning problem assuming that there is no uncertainty in the model parameters. We compare the computational results of the various MINLP and MILP models proposed in the respective section (see Gupta and Grossmann, 2011 for more details).

We consider 10 oil fields (Figure 8) that can be connected to 3 FPSOs with 23 possible connections. There are a total of 84 wells that can be drilled in all of these 10 fields, and the planning horizon considered is 20 years, which is discretized into 20 periods of each 1 year of duration. We need to determine which of the FPSO facilities is to be installed or expanded, in what time period, and what should be its capacity of oil, liquid and gas, to which fields it should be connected and at what time, and the number of wells to be drilled in each field during each time period. Other than these installation decisions, there are operating decisions involving the flowrate of oil, water and gas from each field in each time period. The objective function is to maximize total NPV over the given planning horizon.

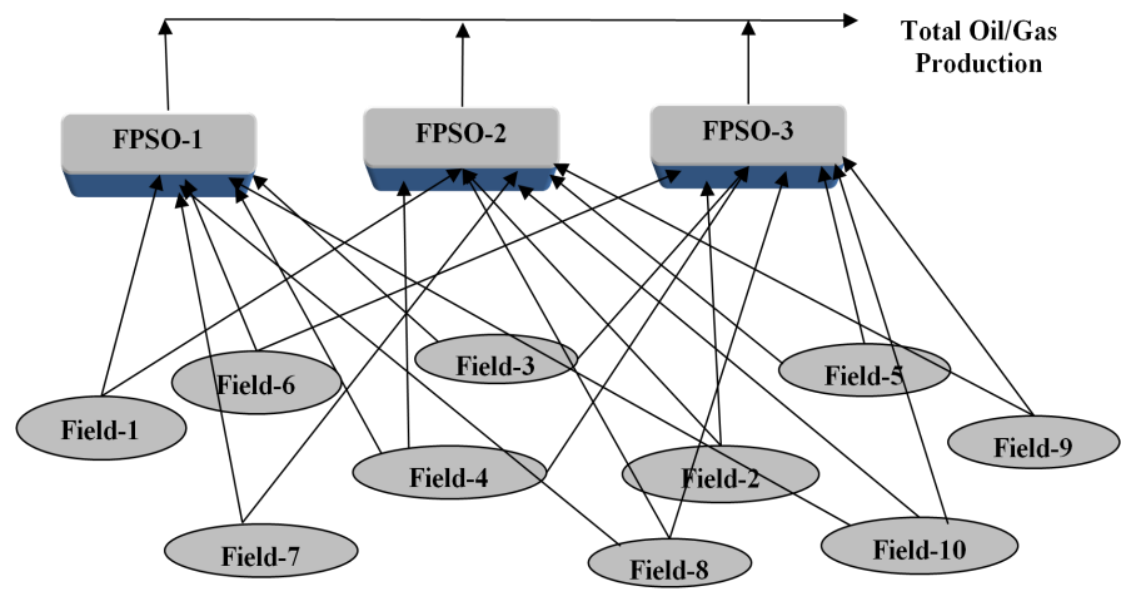

Figure 8: Instance 3 (10 Fields, 3 FPSO, 20 years) for oilfield problem 
The problem is solved using the DICOPT 2x-C solver for MINLP Models 1 and 2, and CPLEX 12.2 for MILP Model 3. These models were implemented in GAMS 23.6.3 and run on Intel Core 17 machine. The optimal solution of this problem that corresponds to reduced MINLP Model 2-R solved with DICOPT 2x-C, suggests to install all the 3 FPSO facilities in the first time period with their respective liquid (Figure 9-a) and gas (Figure 9-b) capacities. These FPSO facilities are further expanded in future when more fields come online or liquid/gas flow rates increases as can be seen from these figures.

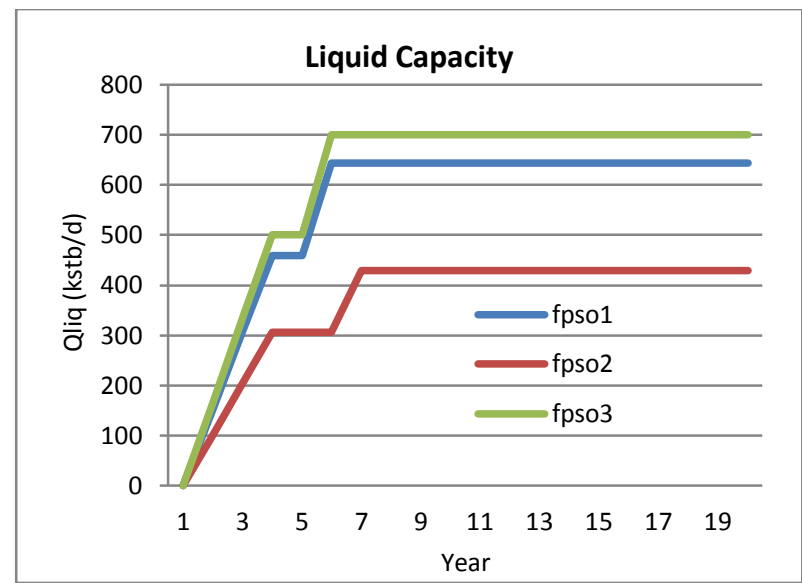

(a) Liquid capacities of FPSO facilities

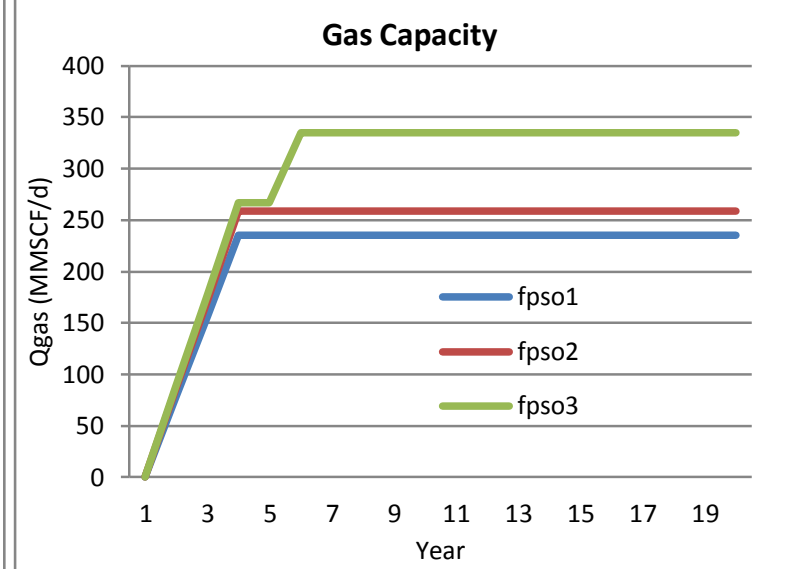

(b) Gas capacities of FPSO facilities

Figure 9: FPSO installation and expansion schedule

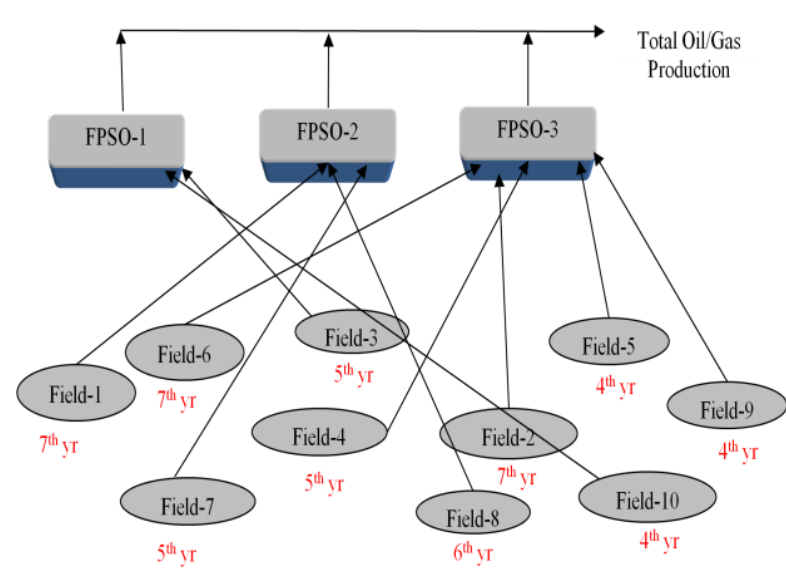

Figure 10: FPSO-field connection schedule

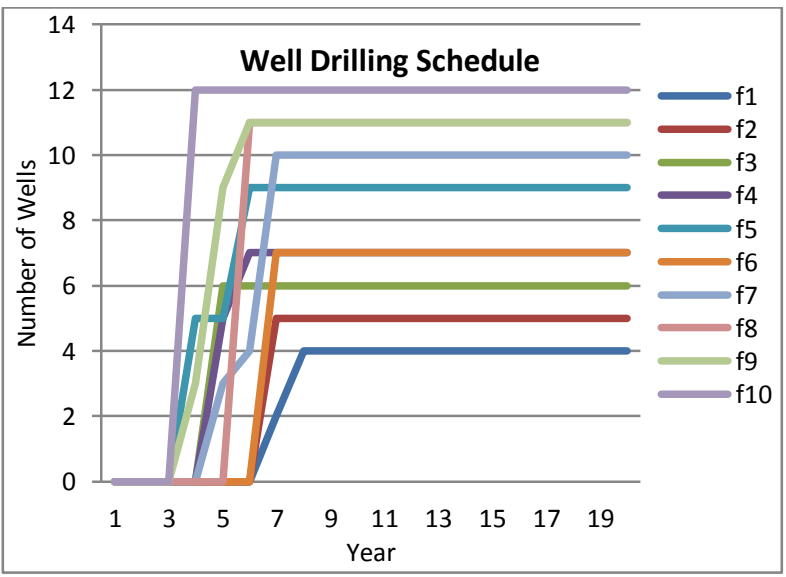

Figure 11: Well drilling schedule for fields

After initial installation of the FPSO facilities by the end of time period 3, these are connected to the various fields to produce oil in their respective time periods for coming online as indicated in Figure 10. The well installation schedule for these fields (Figure 11) ensures that the maximum number of wells drilling limit and maximum potential wells in a field are not 
violated in each time period t. We can observe from these results that most of the installation and expansions are in the first few time periods of the planning horizon. The total NPV of the project is $\$ 30946.39 \mathrm{M}$.

Tables 2-3 represent the results for the various model types considered for this instance. DICOPT performs best in terms of solution time and quality, even for the largest instance compared to other solvers as can be seen from Table 2. There are significant computational savings with the reduced models as compared to the original ones for all the model types in Table 3. Even after binary reduction of the reformulated MILP, Model 3-R becomes expensive to solve, but yields global solutions, and provides a good discrete solution to be fixed/initialized in the MINLPs for finding better solutions.

Table 2: Comparison of various models and solvers for Instance 1

\begin{tabular}{|l|c|c|c|c|}
\hline & \multicolumn{2}{|c|}{ Model 1 } & \multicolumn{2}{c|}{ Model 2 } \\
\hline Constraints & \multicolumn{2}{|c|}{5,900} & \multicolumn{2}{c|}{10,100} \\
\hline Continuous Var. & \multicolumn{2}{|c|}{4,681} & \multicolumn{2}{c|}{6,121} \\
\hline Discrete Var. & \multicolumn{2}{|c|}{ Time (s) } & $\begin{array}{c}\text { Optimal NPV } \\
\text { (million\$) }\end{array}$ & Time (s) \\
\hline Solver & $\begin{array}{c}\text { Optimal NPV } \\
\text { (million\$) }\end{array}$ & 132.34 & 30562.95 & 114.51 \\
\hline DICOPT & 31297.94 & 4973.94 & 30005.33 & 18152.03 \\
\hline SBB & 30466.36 & $>72,000$ & 30562.95 & $>72,000$ \\
\hline BARON & 31297.94 & & & \\
\hline
\end{tabular}

Table 3: Comparison of models 1, 2 and 3 with and w/o binary reduction

\begin{tabular}{|l|c|c|c|c|c|}
\hline & Model 1 & Model 1-R & Model 2 & Model 2-R & Model 3-R \\
\hline Constraints & 5,900 & 5,677 & 10,100 & 9,877 & 17,140 \\
\hline Continuous Var. & 4,681 & 4,244 & 6,121 & 5,684 & 12,007 \\
\hline Discrete Var. & 851 & 483 & 851 & 483 & 863 \\
\hline SOS1 Var. & 0 & 0 & 0 & 0 & 800 \\
\hline NPV(million\$) & 31297.94 & 30982.42 & 30562.95 & 30946.39 & 30986.22 \\
\hline Time(s) & 132.34 & 53.08 & 114.51 & 67.66 & 16295.26 \\
\hline
\end{tabular}

*Model 1 and 2 solved with DICOPT 2x-C, Model 3 with CPLEX 12.2

We can see from Table 4 that the solutions from the Models 1 and 2 after fixing discrete variables based on the MILP solution (even though it was solved within $10 \%$ of optimality tolerance) are the best among all other solutions obtained in Table 2. Therefore, the MILP approximation is an effective way to obtain near optimal solution for the original problem. Notice also that the optimal discrete decisions for Models 1 and 2 are very similar even though 
they are formulated in a different way. However, only Model 2 can be reformulated into an MILP problem that gives a good estimate of the near optimal decisions for these MINLPs.

Table 4: Improved solutions (NPV in million\$) for Models 1 and 2 using Model 3-R solution

\begin{tabular}{|c|c|c|c|}
\hline Model 1 & $\begin{array}{c}\text { Model 1 (fixed } \\
\text { binaries from } \\
\text { Model 3-R) }\end{array}$ & Model 2 & $\begin{array}{c}\text { Model 2 (fixed } \\
\text { binaries from } \\
\text { Model 3-R) }\end{array}$ \\
\hline 31297.94 & 31329.8136 & 30562.95 & 31022.4813 \\
\hline
\end{tabular}

\section{(b) Instance 2: Stochastic Case}

In this example, we consider the planning of offshore oilfield under decision-dependent uncertainty which resolves gradually as a function of investment and operations decisions.

A single reservoir (Figure 12) for 10 years planning horizon is considered that involves decisions about the number, capacity, and installation schedule of FPSO/TLP facilities; the number and drilling schedule of subsea/TLP wells; and the oil production profile over time. The objective is to maximize the expected NPV value. The problem data, details and solution methods are reported in Tarhan et al. (2009).

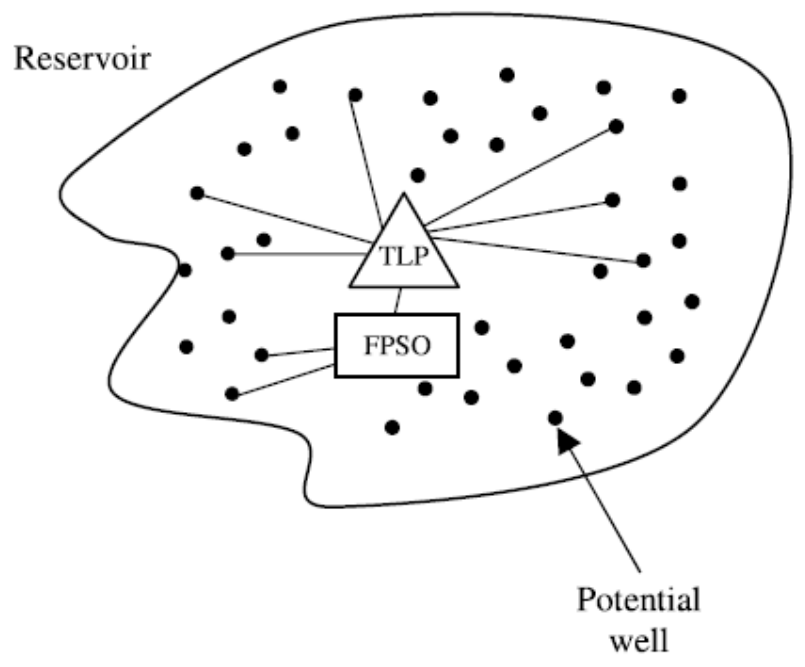

Figure 12: Offshore oilfield Infrastructure for instance 2

The uncertainties in the initial maximum oil flowrate, the size of the reservoirs, and the water breakthrough time are represented by discrete distributions consisting of high and low values resulting in eight scenarios (Table 5). 
Table 5: Scenario representation for example 2

\begin{tabular}{|c|c|c|c|}
\hline Scenarios & $\begin{array}{c}\text { Initial } \\
\text { Productivity per } \\
\text { well (kbd) }\end{array}$ & Reservoir Size & $\begin{array}{c}\text { Water } \\
\text { breakthrough } \\
\text { time }\end{array}$ \\
\hline 1 & 10 & 300 & 5 \\
\hline 2 & 10 & 300 & 2 \\
\hline 3 & 20 & 300 & 5 \\
\hline 4 & 20 & 300 & 2 \\
\hline 5 & 10 & 1500 & 5 \\
\hline 6 & 10 & 1500 & 2 \\
\hline 7 & 20 & 1500 & 5 \\
\hline 8 & 20 & 1500 & 2 \\
\hline
\end{tabular}

The specific rules used for describing the uncertainty resolution are as follows:

The appraisal program is completed when a total of three wells are drilled in one reservoir, which not only gives the actual value for the initial maximum oil flowrate, but also provides the posterior probabilities of reservoir sizes depending on the outcome. The uncertainty in reservoir size can be resolved if either a total of nine or more wells are drilled, or production is made from that reservoir for a duration of 1 year. Uncertainty in the water breakthrough time is resolved after 1 year of production from the reservoir.

The comparison of model statistics given in the paper shows that the size of the full space model increases exponentially as a result of the increase in the number of binary variables for representing uncertainty resolution and the nonanticipativity constraints that relate the decisions in indistinguishable scenarios.

The proposed branch-and-bound algorithm in Tarhan et al. (2009) required $23 \mathrm{~h}$ because, at each node, 40 MINLP problems were solved to global optimality. A total of seven nodes were traversed, and the best feasible solution was found at node 5 . The computational efficiency of the method is further improved by combining global optimization and outer-approximation within proposed duality based branch and bound based algorithm (Tarhan et al. 2011).

The expected value solution proposes building five small FPSO and two TLP facilities and drilling nine subsea wells in the first year. These decisions resolve the uncertainty in the initial productivity and reservoir size. Depending on the values of the reservoir size and initial productivity, different decisions are implemented. This expected value approach gives an objective function value of $\$ 5.81 \times 10^{9}$. The optimal stochastic programming solution yields an 
expected net present value of $\$ 6.37 \times 10^{9}$, which is higher than the expected value solution $\left(\$ 5.81 \times 10^{9}\right)$. The multistage stochastic programming solution proposes building two small FPSO and one TLP facility, and drilling nine subsea wells in the first year. Uncertainty in the initial oil flowrate and reservoir size is resolved after nine wells have been drilled. For scenarios 5 and 6, the solution proposes building four more small FPSO facilities, one large FPSO facility, and five TLP facilities and drilling 12 subsea and three TLP wells. For scenarios 7 and 8, the solution proposes building six more small FPSO facilities and one TLP facility and drilling six subsea wells.

The added value of stochastic programming is due to the conservative initial investment strategy compared to the expected value solution strategy. The stochastic programming approach considers all eight scenarios before making the initial investment. Therefore, it proposes building two small FPSO facilities instead of five and one TLP facility instead of two. Also, it builds more facilities and drills wells only after it determines that the reservoir size is $1500 \mathrm{Mbbl}$. A comparison of net present values of the expected value solution and stochastic programming shows that the added value of stochastic programming comes from handling the downside risk much better than the expected value solution.

\section{(c) Instance 3: Development planning with Complex Fiscal Rules}

Optimal investment and operations decisions, and the computational impact of adding a typical progressive Production Sharing Agreement (PSA) terms for a deterministic case of the oilfield planning problem is demonstrated here.

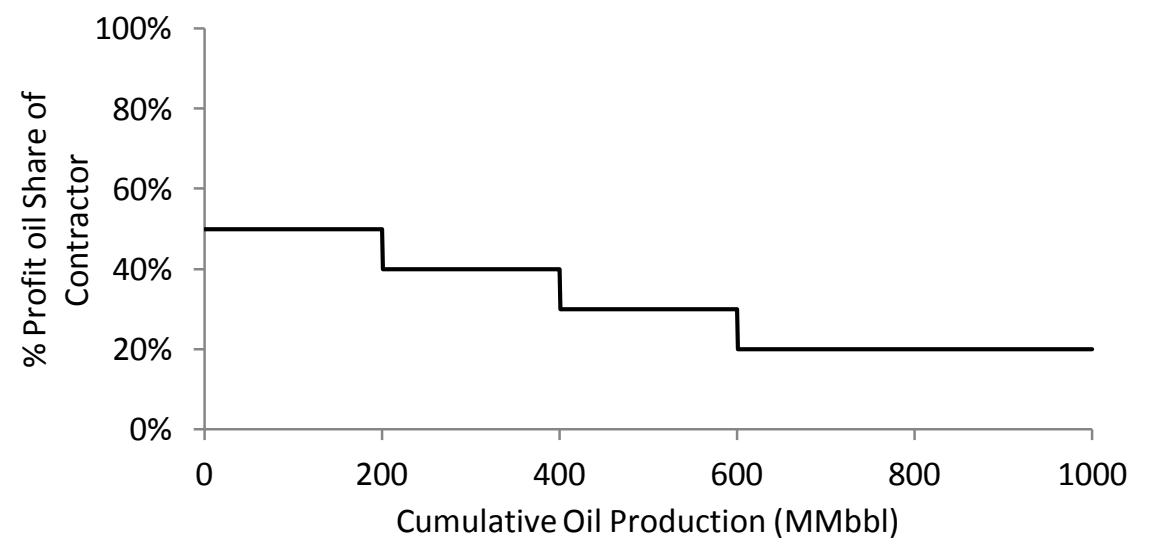

Figure 13. Contractor's Profit oil share for Example 3 
In this instance, we consider 5 oilfields that can be connected to 3 FPSO's with 11 possible connections (Gupta and Grossmann, 2012). There are a total of 31 wells that can be drilled in the 5 fields, and the planning horizon considered is 20 years. There is a cost recovery ceiling and 4 tiers (see. Fig. 13) for profit oil split between the contractor and host Government that are linked to cumulative oil production, which defines the fiscal terms of a typical progressive Production Sharing Agreement.

Table 6 compares the results of the proposed MILP (Model 3) and reduced MILP models (Model 3-R) with progressive PSAs for this example. We can observe that there is significant increase in the computational time with fiscal consideration for the original MILP formulation (Model 3), which takes more than 10 hours with a $14 \%$ of optimality gap as compared to the reduced MILP model (Model 3-R), which terminates the search with a 2\% gap in reasonable time. In contrast, Model 3-R without any fiscal terms can be solved in 189.8 seconds. Therefore, including fiscal rules within development planning can make the problem much harder to solve due to the additional binary variables that are required to model tiers, and resulting weak relaxation.

Note that on contrary the fiscal terms without tier structure, for instance fixed percentage of profit share, royalty rates, may reduce the computational expense of solving the deterministic model directly without any fiscal terms instead. Surprisingly, the problem with flat $35 \%$ of the profit share of contractor is solved in $72.64 \mathrm{~s}$ which is even smaller than the solution time for deterministic case without any fiscal terms (189.8s). On the other hand, the problem with 2 tiers instead of 4 as considered above is solved in $693.71 \mathrm{~s}$ which is more than the model without fiscal terms and less than the model with 4 tiers. Therefore, the increase in computational time while including fiscal rules within development planning is directly related to the number of tiers (steps) that are present in the model to determine the profit oil shares or royalties.

Table 6. Computational Results for Example 3

\begin{tabular}{|c|c|c|c|c|c|}
\hline Model & Constraints & $\begin{array}{c}\text { Continuous } \\
\text { Variables }\end{array}$ & $\begin{array}{c}\text { Discrete } \\
\text { Variables }\end{array}$ & $\begin{array}{c}\text { NPV } \\
\text { (\$Million) }\end{array}$ & Time (s) \\
\hline Model 3 with PSA & 9474 & 6432 & 727 & $2,183.63$ & $>36,000$ \\
\hline Model 3-R with PSA & 9363 & 6223 & 551 & $2,228.94$ & $1,163.7$ \\
\hline
\end{tabular}

The optimal solution from Model 3-R with fiscal considerations suggests installing 1 FPSO facility with expansions in the future (see Fig. 14), while Fig. 15 represents the well drilling schedule for this example. The tiers 2, 3 and 4 for profit oil split become active in years 6,8 and 
12, respectively, based on the cumulative oil production profile during the given planning horizon.

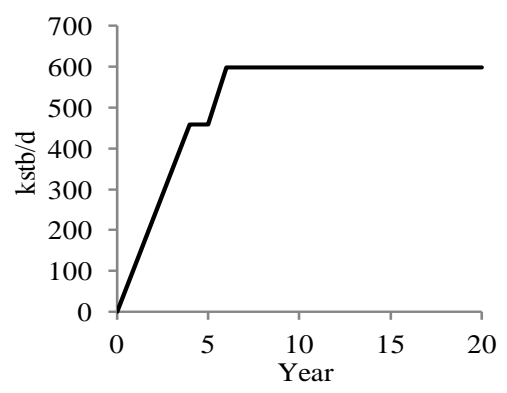

(a) Liquid capacity

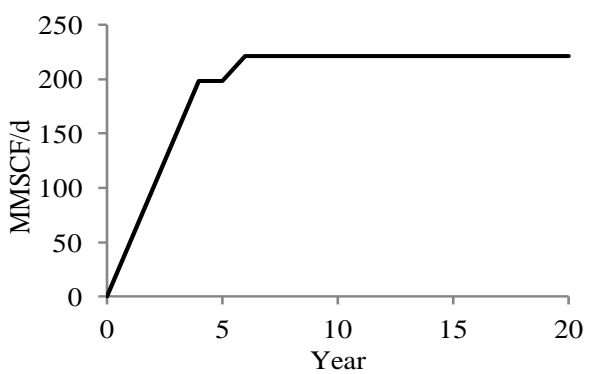

(b) Gas capacity

Figure 14. Liquid and Gas capacities of FPSO 3

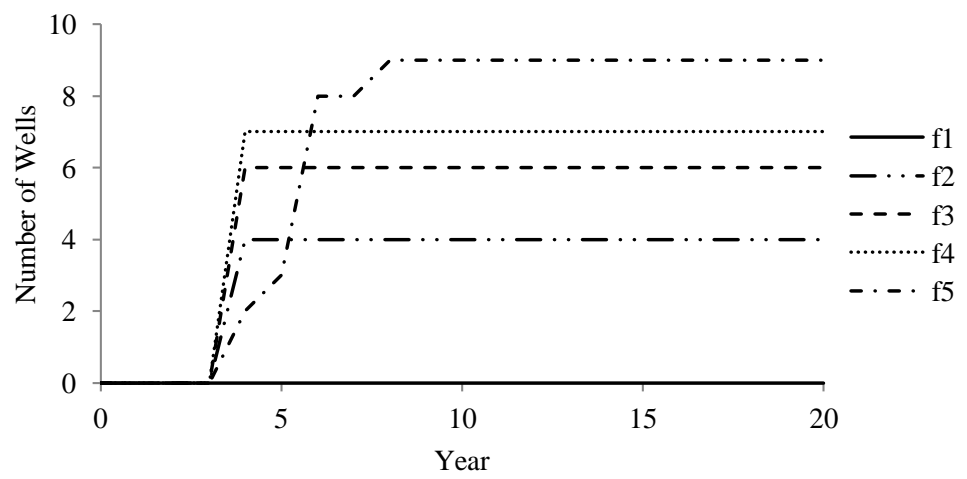

Figure 15. Well drilling schedule for Example 3

\section{Conclusions}

In this paper, we have first reviewed a new generic model for offshore oil and gas field infrastructure investment and operational planning considering multiple fields, three components (oil, water and gas), facility expansions decisions, well drilling schedules and nonlinear reservoir profiles. The detailed model and its reformulations to improve the computational efficiency have been discussed. Furthermore, to address the issue of uncertainty in key model parameters, recent work on multiage stochastic programming based approaches was highlighted. Discussion on the extensions of the proposed deterministic model to incorporate uncertainties and generic fiscal rules in a unified framework, and possible complications was presented. Numerical results on development planning problems involving perfect information or uncertainty were reported, as well as the handling of fiscal considerations, specifically Production Sharing Agreements. It is 
hoped that this paper has shown that there has been very significant progress in the mathematical programming models for offshore development planning.

\section{References}

1. Ahmed, S., 2000. Strategic planning under uncertainty: Stochastic integer programming approaches. Ph.D. thesis, University of Illinois at Urbana-Champaign.

2. Aronofsky JS, Williams AC. The use of linear programming and mathematical models in underground oil production. Manage Sci. 1962; 8:394-407.

3. Aseeri, A., Gorman, P., Bagajewicz, M. J. Financial risk management in offshore oil infrastructure planning and scheduling. Ind. Eng. Chem. Res. 2004, 43, 3063-3072.

4. Babusiaux, D., Favennec, J. P., Bauquis, P. R., Bret-Rouzaut, N., and Guirauden, D. (2007). Oil and Gas Exploration and Production: Reserves, costs, contracts. Technip edition, 2007, chap. 4.

5. Bailey, W. J.; Couet, B.; Wilkinson, D. Field Optimization Tool for Maximizing Asset Value. SPE ReserVoir EVal. Eng. 2005, 8 (1), 7-21.

6. Barnes, R., Linke, P., Kokossis, A. Optimization of oil-field development production capacity. European symposium on computer aided process engineering, 2002, vol. 12 (p. 631).

7. Barnes, R.J., Kokossis, A. and Shang, Z. An integrated mathematical programming approach for the design and optimisation of offshore fields. Computers and Chemical Engineering, 31, Issues 5-6, 2007, 612-629.

8. Begg, S. H., Bratvold, R., Campbell, J. C. Improving Investment Decisions Using A Stochastic Integrated Asset Model Presented at the SPE Annual Technical Conference and Exhibition, New Orleans, LA, Sep 30-Oct 3, 2001; Paper SPE 71414.

9. Behrenbruch, P. Offshore oilfield development planning. J. Pet. Technol. 1993, 45 (8), 735-743.

10. Birge, J. R., Louveaux, F., 1997. Introduction to stochastic programming. New York, NY: Springer.

11. Bohannon J. A linear programming model for optimum development of multi-reservoir pipeline systems. J Petrol Tech. 1970;22:1429-1436.

12. Boland, N., Dumitrescu, I., Froyland, G., 2008. A multistage stochastic programming approach to open pit mine production scheduling with uncertain geology. http://www.optimizationonline.org/DBHTML/2008/10/2123.html.

13. BP Statistical Review of World Energy, 2011.

14. Caroe, C.C., Schultz, R., 1999: Dual decomposition in stochastic integer programming. Oper. Res. Lett. 24, 37-45.

15. Carvalho, M., Pinto, J. M. A bilevel decomposition technique for the optimal planning of offshore platforms. Brazilian J. Chem. Eng. 2006a, 23, 67-82. 
16. Carvalho, M., Pinto, J. M. An MILP model and solution technique for the planning of infrastructure in offshore oilfields. J. Pet. Sci. Eng. 2006b,51, 97-110.

17. Clay, R. L., Grossmann, I. E., 1997. A disaggregation algorithm for the optimization of stochastic planning models. Computers and Chemical Engineering 21 (7),751-774.

18. Colvin, M., Maravelias, C. T., 2008. A stochastic programming approach for clinical trial planning in new drug development. Computers and Chemical Engineering 32 (2008) 2626-2642

19. Colvin, M., Maravelias, C.T., 2010. Modeling methods and a branch and cut algorithm for Pharmaceutical Clinical Trial Planning using Stochastic Programming, European Journal of Operational Research, 203 (2010) 205-215.

20. Cullick, A. S., Heath D., Narayanan K., April J., Kelly J. Optimizing Multiple-Field Scheduling and Production Strategy with Reduced Risk. Presented at the SPE Annual Technical Conference and Exhibition, Denver, CO, Oct 5-8, 2003; Paper SPE 84239.

21. Cullick, A. S., Cude, R., and Tarman, M. 2007. Optimizing Field Development Concepts for Complex Offshore Production Systems, Paper SPE 108562-MS presented at Offshore Europe, Aberdeen, Scotland, U.K., 4-7 September.

22. Dias, M. A. G., 2004. Valuation of exploration and production assets: an overview of real options models. Journal of Petroleum Science and Engineering 44 (1-2), 93-114.

23. Elgsæter, S. M., O. Slupphaug, Johansen T. A. A structured approach to optimizing offshore oil and gas production with uncertain models. Comput. Chem. Eng. 2010, 34(2), 163-176.

24. Ettehad, A., Jablonowski, C. J., Lake, L. W. Stochastic Optimization and Uncertainty Analysis for E\&P Projects: A Case in Offshore Gas Field Development, Offshore Technology Conference, 2011.

25. Goel, V., Grossmann, I. E. A stochastic programming approach to planning of offshore gas field developments under uncertainty in reserves. Comput. Chem. Eng. 2004, 28 (8), 1409-1429.

26. Goel, V., Grossmann, I. E. A class of stochastic programs with decision dependent uncertainty. Mathematical Programming, 2006, 108(2-3, Ser. B), 355-394.

27. Goel, V., Grossmann, I. E., El-Bakry, A. S., Mulkay, E. L. A novel branch and bound algorithm for optimal development of gas fields under uncertainty in reserves. Comput. Chem. Eng. 2006, 30, 1076-1092.

28. Grothey, A., McKinnon, K., 2000. Decomposing the optimization of a gas lifted oil well network. Tech Rept. MS 00-005, Department of Mathematics and Statistics, University of Edinburgh.

29. Gunnerud, V., Foss, B. Oil production optimization - A piecewise linear model, solved with two decomposition strategies. Computers and Chemical Engineering, 2010, 34, 1803-1812.

30. Gupta, V., Grossmann, I. E. Solution strategies for multistage stochastic programming with endogenous uncertainties. Comput. Chem. Eng. 2011, 35, 2235-2247. 
31. Gupta, V., Grossmann, I. E. An Efficient Multiperiod MINLP Model for Optimal Planning of Offshore Oil and Gas Field Infrastructure. I\&EC Research, 2011, Submitted for publication.

32. Gupta, V., Grossmann, I. E. Optimal Development Planning of Offshore Oil and Gas Field Infrastructure under Complex Fiscal Rules. Proceedings of Foundations of Computer-Aided Process Operations (FOCAPO 2012), In press.

33. Gupta, V., Grossmann, I. E. Modeling and Solution strategies for Offshore Oilfield Development Planning under Complex Fiscal Rules. Working paper, 2012.

34. Haugen, K. K., 1996. A stochastic dynamic programming model for scheduling of offshore petroleum fields with resource uncertainty. European Journal of Operational Research 88 (1), 88-100.

35. Haugland, D., Hallefjord, Å.; Asheim, H. Models for Petroleum Field Exploitation. Eur. J. Oper. Res. 1988, 37, 58.

36. Held, H., Woodruff, D. L., 2005. Heuristics for multi-stage interdiction of stochastic networks. Journal of Heuristics 11 (5-6), 483-500.

37. Ierapetritou, M. G., Pistikopoulos, E. N., 1994. A novel optimization approach of stochastic planning models. Industrial and Engineering Chemistry Research 33, 19301942.

38. Iyer, R. R., Grossmann, I. E., 1998. A bilevel decomposition algorithm for long-range planning of process networks. Industrial and Engineering Chemistry Research 37 (2), 474-481.

39. Iyer, R. R., Grossmann, I. E., Vasantharajan, S., Cullick, A. S. Optimal planning and scheduling offshore oilfield infrastructure investment and operations. Ind. Eng. Chem. Res. 1998, 37, 1380-1397.

40. Jonsbraten, T. W. Oil-field optimization under price uncertainty. Journal of the Operational Research Society, 1998, 49, 811.

41. Jonsbraten, T., 1998. Optimization models for petroleum field exploitation. PhD thesis, Norwegian School of Economics and Business Administration.

42. Jonsbraten, T. W.; Wets, R. J. B.; Woodruff, D. L. A class of stochastic programs with decision dependent random elements. Ann. Oper. Res. 1998, 82, 83-106.

43. Kaiser, M.J. and A.G. Pulsipher. Fiscal System Analysis: Concessionary and Contractual Systems Used in Offshore Petroleum Arrangements. US Department of the Interior Mineral Management Services MMS 2004-016, 1-78.

44. Kosmidis, V., Perkins, J., Pistikopoulos, E., 2002. A mixed integer optimization strategy for integrated gas/oil production. In: European Symposium on Computer Aided Process Engineering. Vol. 12.

45. Kosmidis, V. D.; Perkins, J. D.; Pistikopoulos, E. N. Optimization of Well Oil Rate Allocations in Petroleum Fields. Ind. Eng. Chem. Res. 2004, 43, 3513. 
46. Kosmidis, V. D., Perkins, J. D., Pistikopoulos, E. N. A mixed integer optimization for the well scheduling problem on petroleum fields. Computers \& Chemical Engineering Journal, 2005, 29, 1523-1541.

47. Lee AS, Aronofsky JS. A linear programming model for scheduling crude oil production. J Petrol Tech. 1958;10:51-54.

48. Lee, S., Grossmann, I. E. New algorithms for nonlinear generalized disjunctive programming. Computers and Chemical Engineering 2000, 24 (9-10), 2125-2141.

49. Ligero, E. L., Xavier, A. M., Schiozer, D. J. Value of information during appraisal and development of petroleum fields. Presented at the $18^{\text {th }}$ International Congress of Mechanical Engineering, Ouro Preto, MG Brasil, Nov 6-11, 2005.

50. Lin, X., Floudas, C. A. A Novel Continuous-Time Modeling and Optimization Framework for Well Platform Planning Problems. Optim. Eng. 2003, 4 (1-2), 65-95.

51. Lund, M. W. 1999. Real options in offshore oil field development projects, Proceedings $3^{\text {rd }}$ International Conference on Real Options, 6-8 June Wassenaar, Holland.

52. Lund, M. W. Valuing Flexibility in Offshore Petroleum Projects. Ann. Oper. Res. 2000, 99 (1-4), 325-349.

53. Lund, D., 2005. How to analyze the investment-uncertainty relationship in real options models? Review of Financial Economics 14, 311-322.

54. Ortiz-Gomez, A., Rico-Ramirez, V., \& Hernandez-Castro, S. Mixed-integer multiperiod model for the planning of oil-field production. Comput. Chem. Eng., 2002, 26(4-5), 703.

55. Ozdogan, U.; Horne, R. N. Optimization of well placement under time-dependent uncertainty. SPE Reservoir Eval. Eng. 2006, 9 (2), 135-145.

56. Pflug, G. Ch., 1990. Online optimization of simulated markovian processes. Mathematics of Operations Research Res. 15, No.3, 381-395.

57. Rico-Ramirez V., Grossmann I. E., Tarhan B., Hernández-Castro S., Segovia-Hernández J. G. An Approach to the Representation of Gradual Uncertainty Resolution in Stochastic Multiperiod Planning. Computer Aided Chemical Engineering, 26, 2009, 713-718.

58. Ruszczynski, A., 1997. Decomposition methods in stochastic programming. Math. Programming (Ser. B) 79, 333-353.

59. Sullivan J. A computer model for planning the development of an offshore gas field. J Petrol Tech. 1982;34:1555-1564.

60. Sahinidis, N.V., 2004. Optimization under uncertainty: State-of-the-art and opportunities. Comput. Chem. Eng. 28 (6-7), 971-983.

61. Schultz, R., 2003. Stochastic programming with integer variables. Mathematical Programming 97 (1-2), 285-309.

62. Solak, S., 2007. Efficient solution procedures for multistage stochastic formulations of two problem classes. PhD thesis, Georgia Institute of Technology.

63. Sunley, E. M., T. Baunsgaard, and D. Simard. 2002. "Revenue from the Oil and Gas Sector: Issues and Country Experience.” IMF Conference Paper. 
64. Tarhan, B., Grossmann, I. E., 2008. A multistage stochastic programming approach with strategies for uncertainty reduction in the synthesis of process networks with uncertain yields. Computers and Chemical Engineering 32, 766-788.

65. Tarhan, B., Grossmann, I.E., Goel, V. Stochastic programming approach for the planning of offshore oil or gas field infrastructure under decision- dependent uncertainty. Ind. Eng. Chem. Res. 2009, 48(6), 3078-3097.

66. Tarhan, B., Grossmann, I.E., Goel, V. Computational strategies for non-convex multistage MINLP models with decision-dependent uncertainty and gradual uncertainty resolution. Ann. Oper. Res., 2011, DOI: 10.1007/s10479-011-0855-x.

67. Tordo, S. 2007. Fiscal Systems of Hydrocarbons: Design Issues. Working Paper 123/07, World Bank, Washington, D.C.

68. Tsarbopoulou, C. Optimization of oil facilities and oil production. M.Sc. Dissertation, University College London, London, U.K., 2000.

69. Ulstein, N. L., Nygreen, B.; Sagli, J. R. Tactical planning of offshore petroleum production. Eur. J. Oper. Res. 2007, 176, 550-564.

70. Viswanath, K., Peeta, S., Salman, S. F., 2004. Investing in the links of a stochastic network to minimize expected shortest path length. Tech. rep., Purdue University.

71. van den Heever, S. A.; Grossmann, I. E. An iterative aggregation/disaggregation approach for the solution of a mixed integer nonlinear oilfield infrastructure planning model. Ind. Eng. Chem. Res. 2000, 39, 1955-1971.

72. van den Heever, S. A., Grossmann, I. E., Vasantharajan, S.; Edwards, K. Integrating complex economic objectives with the design and planning of offshore oilfield infrastructures. Comput. Chem. Eng. 2000, 24, 1049-1055.

73. van den Heever, S. A., Grossmann, I. E. A Lagrangean Decomposition Heuristic for the Design and Planning of Offshore Hydrocarbon Field Infrastructures with Complex Economic Objectives. Ind. Eng. Chem. Res. 2001, 40, 2857-2875.

74. World Bank. 2007. "Contracts for Petroleum Development - Part 1-3." Petroleum Sector Briefing Note No. 7.

75. You F., Wassick J. M., Grossmann I. E. Risk management for global supply chain planning under uncertainty: models and algorithms. AIChE J. 2009, 55, 931-946.

76. Zabalza-Mezghani, I.; Manceau, E.; Feraille, M.; Jourdan, A. Uncertainty management: From geological scenarios to production scheme optimization. J. Pet. Sci. Eng. 2004, 44, $11-25$. 